\title{
Avaliação do tratamento da coledocolitíase residual
}

Dissertação apresentada à Faculdade de Medicina da Universidade de São Paulo para a obtenção do título de Mestre em Ciências.

Área de concentração: Cirurgia do Aparelho Digestivo

Orientador: Dr. Marco Aurelio Santo

\section{SÃO PAULO}




\begin{abstract}
À
Lezinda e José (in memorian), meus pais, pelo amor, formação e incentivo.
\end{abstract}

Renato, meu filho pela compreensão, e a razão da dedicação a este trabalho. 


\section{AGADECIMENTOS}

\section{Ao Drs Leosarte Alves e Helio Ponciano Trevenzol, amigos e orientadores e sem os quais não seria possível a confecção deste trabalho.}

Aos professores Bruno Zilberstein, Ivan Cecconello, cirurgiões da melhor estirpe e possuidores de atilada inteligência; as palavras gradidão, reconhecimento, admiração e respeito e todos os seus sinônimos encontrados nos dicionários são muito poucos para expressar o que sinto.

À professora Mariza Martins Avelino, infecto-pediatra, pelo carisma, dedicação, incentivo, estímulo incessante desde a época de acadêmico, uma grande incentivadora.

Aos colegas Noboro Sugita, Jhonn Kennedy de Barros, grandes amigos e incentivadores pelo convívio e aprendizado desde a época de residente.

Aos professores Luiz Rassi, Joffre Marcondes Resende, pessoas e mestres em todos os momentos da vida que transmitem conhecimento apenas num simples olhar.

Ao residente Ronney Carvalho e acadêmica Evellim Lorena com suas eficiências e dedicação no levantamento de prontuários.

A auxiliar de enfermagem e instrumentadora Josefa Selma pela dedicação e compreensão no relacionamento dia a dia durante a confecção deste trabalho.

Aos amigos, colegas mestrandos e chefes do Departamento de Cirurgia do Hospital das Clínicas da Universidade Federal de Goiás pelo incentivo a cada passo durante a realização do presente estudo.

Ao amigo mestrando Marcus Vinicius S. Nei pela participação neste trabalho na realização dos exames e procedimentos endoscópicos.

À administração do Hospital das Clínicas da Faculdade de Medicina da Universidade Federal de Goiás por ter me acolhido com carinho e apreço nas dependências desta instituição.

Ao professor Joaquim Gama Rodrigues, professor titular da Faculdade de Medicina da Universidade de São Paulo, por ter proporcionado a viabilização deste Programa Inter-institucional. 


\section{SUMÁRIO}

Lista de Abreviaturas

Lista de Figuras

Lista de Tabela

Resumo

Summary

1. INTRODUÇÃO

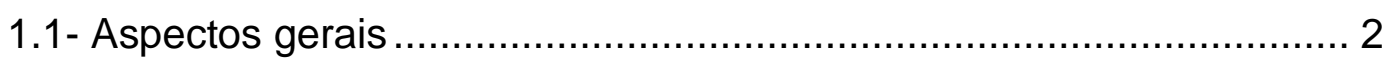

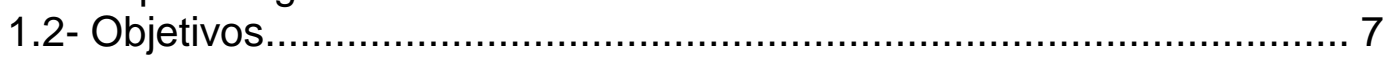

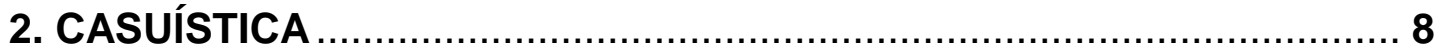

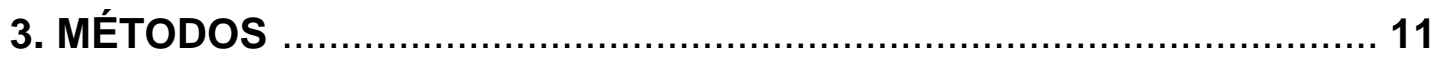

3.1- Avaliação diagnóstica de coledocolitíase residual ........................... 12

3.1.1- Critérios clínicos .....................................................................12

3.1.2- Análise laboratorial ......................................................................12

3.1.3- Métodos de imagem ....................................................................13

3.1.3.1- Ultra-sonografia ............................................................13

3.1.3.2- Colangiografia pelo dreno de Kehr..........................................14

3.1.3.3- Colangio-pancreatografia retrógrada ……………...................14

3.1.3.4- Ecoendoscopia ...........................................................15

3.1.3.5- Colangio-ressonância magnética ............................................16

3.2- Procedimento terapêutico utilizado............................................... 16

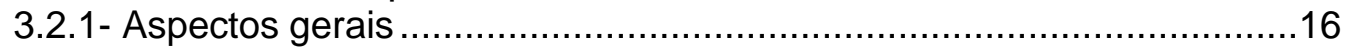

3.2.2- Papilotomia endoscópica ................................................................17

3.2.3- Procedimento cirúrgico ……………………………………....... 18

3.2.3.1- Coledocolitomia com papilotomia trans-duodenal .....................18

3.2.3.2- Derivação bílio-digestiva em Y-de-Roux ....................................19

3.2.3.3- Derivação colédoco-duodenal.................................................20

3.3- Sucesso e insucesso no clareamento ……………....................... 21

3.4- Complicações ...................................................................... 21

3.4.1- Complicações endoscópicas …………………………..................21

3.4.2- Complicações cirúrgicas ………………………………………......22

3.5- Período de internação pós-operatório …………………………... 22

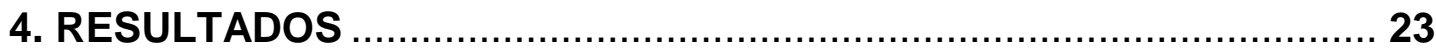

4.1- Avaliação diagnóstica de coledocolitíase residual ............................ 24

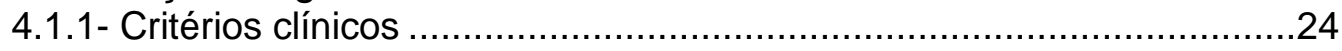

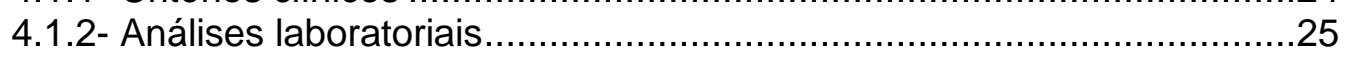

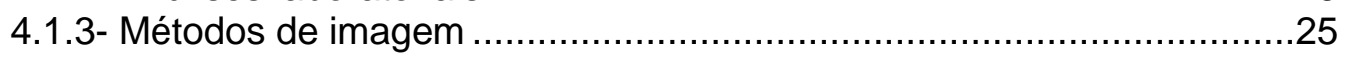

4.1.3.1- Ultra-sonografia ............................................................2

4.1.3.2- Colangiografia pelo dreno Kehr..............................................26

4.1.3.3- Colangiopancreatografia retrógrada.......................................26

4.1.3.4- Ecoendoscopia ....................................................................26

4.1.3.5- Colangioressonância magnética ............................................26 


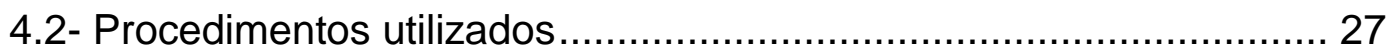

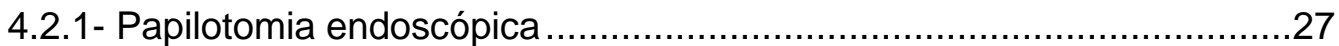

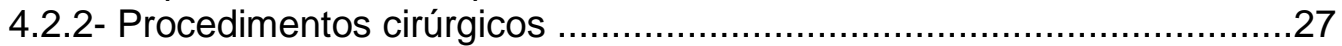

4.2.2.1- Coledocolitomia com papilotomia trans-duodenal ....................27

4.2.2.2- Derivação bílio-digestiva em Y-de-Roux ..................................27

4.2.2.3- Derivação colédoco-duodenal...............................................28

4.3- Sucesso e insucesso no clareamento ........................................ 28

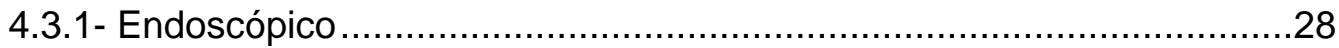

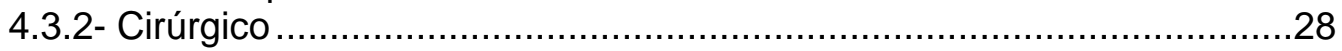

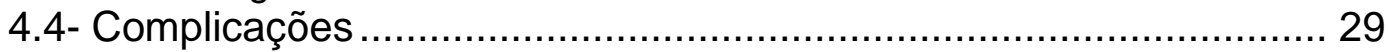

4.5- Período de internação .............................................................. 30

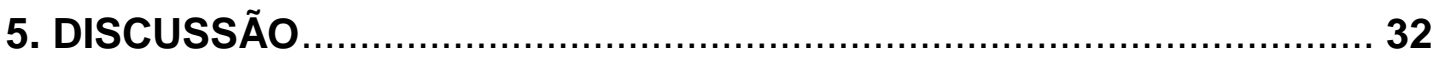

5.1- Avaliação diagnóstica da coledocolitíase residual......................... 34

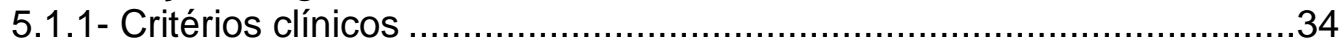

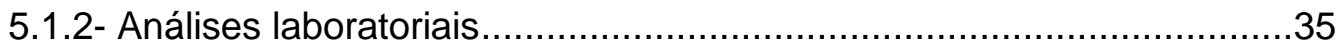

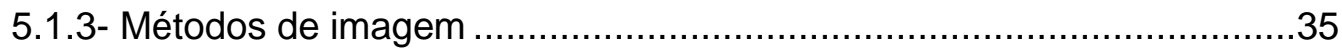

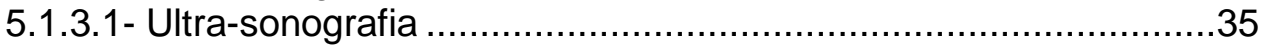

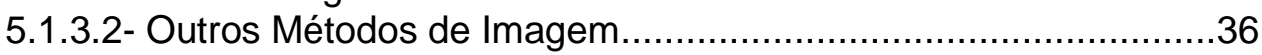

5.1.3.2a- Colangiografia pelo Kehr.................................................36

5.1.3.2b-Colangiopancreatografia retrógrada....................................37

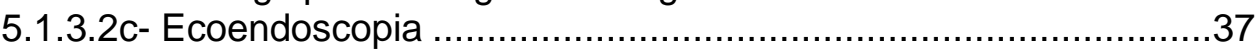

5.1.3.2d- Colangiorressonância magnética .........................................37

5.2- Procedimentos utilizados.......................................................... 38

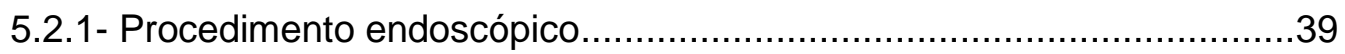

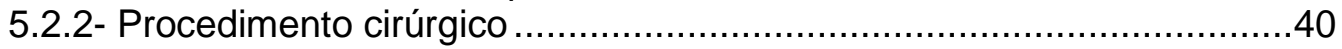

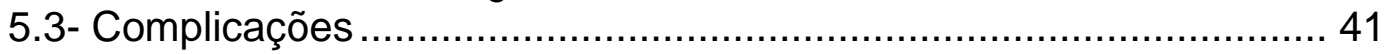

5.4- Período de internação pós-operatório ....................................... 42

6. CONCLUSÃO

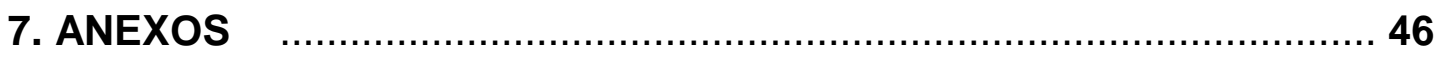

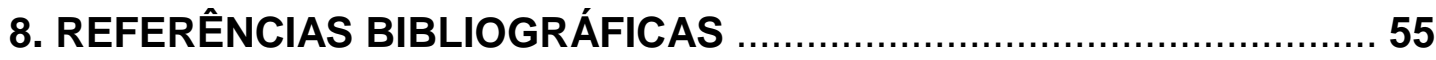




\section{LISTA DE FIGURAS}

Figura 01- Ultra-sonografia nos dois primeiros diapositivos mostra apenas dilatação coledociana e na foto inferior a presença de cálculo no seu interior com a presença de dilatação de hepato-colédoco.

Figura 2- Colangiografia pelo dreno de Kehr onde podemos ver a presença de cálculo no interior da via biliar dilatada.

Figura 3 - Colangiopancreatografia retrógrada onde podemos ver a presença de colédoco dilatado com cálculo no seu interior e também impactado em papila duodenal.

Figura 4-Ecoendoscopia onde mostra a presença de cálculo no interior da via biliar principal dilatada.

Figura 5 - Colangio-ressonância magnética onde mostra a presença de cálculo impactado em colédoco terminal com dilatação de via biliar principal.

Figura 6 - Papilotomia onde podemos ver a papila já aberta com clareamento da via biliar principal com "Basket" e cálculo na luz intestinal.

Figura 7 - Papilotomia trans-duodenal onde podemos ver a abertura do duodeno como identificar a papila com a realização da papilotomia.

Figura 8 - Derivação em Y-de-Roux onde mostra a confecção transmesocólica.

Figura 9 - Derivação colédoco-duodenal onde mostra a sua confecção latero-lateral em colédoco dilatado. 


\section{LISTA DE TABELAS}

TABELA 1 - Critérios clínicos 24

TABELA 2 - Análises laboratoriais - exames alterados ........................... 25

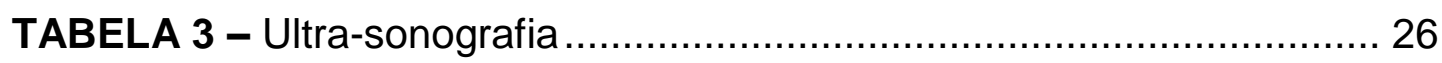

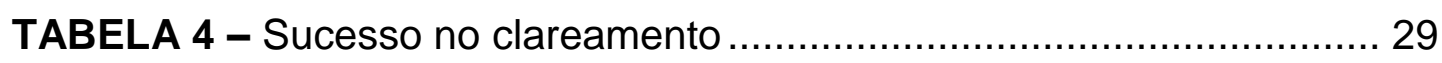

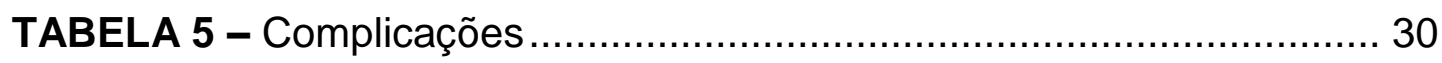

TABELA 6 - Período de internação em dias pós-procedimento .................. 31 


\section{RESUMO}

FERREIRA, BD. Avaliação do tratamento da coledocolitíase residual. São Paulo, 2003, Dissertação (Mestrado) - Faculdade de Medicina, Universidade de São Paulo.

A coledocolitíase residual representa grande desafio na avaliação diagnóstica e proposta terapêutica. Neste contexto, realizamos estudo retrospectivo com o objetivo de avaliar critérios clínicos, laboratoriais e métodos de imagem para o seu diagnóstico; avaliar o resultado do tratamento através de procedimentos endoscópicos e cirúrgicos, bem como a ocorrência de complicações e sua repercussão no período de internação. Foram estudados 32 (trinta e dois) pacientes portadores de coledocolitíase residual internados na Clínica Cirúrgica do Hospital das Clínicas da Faculdade de Medicina da Universidade Federal de Goiás, no período de janeiro de 1995 a julho de 2001. Foram incluídos pacientes submetidos previamente a colecistectomia, nos quais o diagnóstico de coledocolitíase foi feito posteriormente (pela não realização de colangiografia trans-operatória) ou no próprio curso da colecistectomia, porém postergando-se o tratamento. Pudemos concluir: a maioria dos pacientes portadores de coledocolitíase residual tem como sintoma principal a icterícia; a ultra-sonografia não é um método diagnóstico eficaz, uma vez que demonstrou alteração de via biliar em cerca de $50 \%$ dos pacientes; tanto o procedimento endoscópico como o cirúrgico mostraram alto índice de clareamento da via biliar (acima de $90 \%$ ), sendo a morbidade baixa e mortalidade nula em ambos os procedimentos; 0 período de internação foi menor quando o procedimento endoscópico foi realizado. 


\section{SUMMARY}

FERREIRA, BD. Treatment evaluation of residual choledocholithiasis. São Paulo, 2003, Dissertação (Mestrado) - Faculdade de Medicina, Universidade de São Paulo.

Choledocholithiasis represents a great challenge in diagnostic evaluation and therapeutics. Because of it we've proposed a retrospective study to analise the clinical and laboratorial criteria and image studies to the diagnosis of such condition. It was possible to evaluate the treatment (endoscopic or surgical) and complications with these information. Thus, with this aim, 32 (thirty-two) patients were evaluated. They were suffering from residual choledocholithiasis and were admitted on the Surgical Unit of the General Hospital of the Medical School of University of Goiás, from january 1995 to july 2001. It was included patients that were performed on a previous cholecistectomy. The diagnose of choledocholithiasis was get either during the surgery, although the definitive treatment had been postponed, or on the follow-up. We concluded that most part of the patients with residual choledocholithiasis were icteric and that the ultrasound study is not an effective method to detect residual choledocholithiasis (positive around $50 \%$ ). Besides, both therapeutic procedures (endoscopic and surgical) were successful in the cleaning of biliary ducts (above 90\%), had low morbidity and no mortality. The discharge of the patient was faster in case of endoscopic procedure. 


\section{1- INTRODUÇÃO}

\section{1- Aspectos gerais}

A doença calculosa do trato biliar é um problema significativo de saúde. Sua prevalência gira em torno de $10 \%$ na população em geral, mas ocorre certa variação relacionada aos costumes, fatores geográficos e afecções pré-existentes. A composição química do cálculo também varia segundo hábitos alimentares e afecções prévias do paciente (NAKAYAMA, KOGA: 1984).

No Brasil, estudo conduzido por COELHO et al. (1999) demonstra prevalência de litíase vesicular em 9,3\% da população adulta. Este índice eleva-se de $2,4 \%$ para $27,5 \%$ quando comparadas as faixas etárias de 20 a 29 anos e mais de 70 anos, respectivamente. Em mulheres acima de 50 anos é superior a $20 \%$.

A coledocolitíase consiste na presença de calculo no hepatocolédoco, podendo determinar sua obstrução parcial ou total. Existem várias formas de manifestação clínica. Sua prevalência varia entre 4 a 14\% dos portadores de colelitíase, sendo que um terço desses casos apresentam-se de forma 
assintomática (BERCI; MORGENSTERN,1994, MILLAT, et al., 1995, SAVASSI-ROCHA, 1997), constituindo-se em uma importante complicação, com várias possibilidades evolutivas para outras complicações mais graves, como pancreatite aguda, icterícia obstrutiva e insuficiência renal aguda. (MONTEIRO DA CUNHA, 1994).

A coledocolitíase pode ser classificada em primária ou secundária. $\mathrm{Na}$ primária, os cálculos são formados na própria via biliar principal, tanto intra quanto extra-hepática (ALLEN; BERNHOFT, 1981, WAY ET AL., 1981). Já na secundária, os cálculos são formados na vesícula biliar, com migração para a via biliar principal, por via trans-cística ou através do desenvolvimento de fístula vesículo-coledociana.

A coledocolitíase residual correlaciona-se ao tratamento cirúrgico da colelitíase, ou seja, a colecistectomia. Define-se como sendo a persistência de cálculo(s) na via biliar principal por falta de diagnóstico pela não realização da colangiografia intra-operatória ou por falha do exame, ou ainda por conduta insuficiente de tratamento, ou seja, diagnosticada e não tratada concomitantemente (GANEY et al., 1986, GARDNER et al., 1966, GERBER, 1982, HAUER-JENSEN et al,1993, LORIMER et al., 1997, McENTEE et al., 1991, MURISON et al.,1996, SCHWESINGER,1997; SANTO.,2000), seja por questões técnicas ou devido à falta de condições clínicas do paciente durante a intervenção cirúrgica.

A suspeita clínica da coledocolitíase é feita através da presença de sinais característicos (dor tipo cólica em andar superior do abdômen, com 
irradiação para o dorso e icterícia). A avaliação diagnóstica se faz através de vários procedimentos complementares:

- Laboratoriais: dosagem sérica da fração direta das bilirrubinas, gama-glutamiltransferase (gama GT) e fosfatase alcalina.

- Avaliações por métodos de imagem: ultra-sonografia; ressonância magnética (colangio-ressonância), colangio-pancreatografia retrógrada e ecoendoscopia (GANC, 1997; SANTO,2000).

Uma vez firmado o diagnóstico, impõe-se o tratamento, que é variável e depende de cada caso. Classicamente para a extração do cálculo realizase a coledocotomia com ou sem papilotomia trans-duodenal, seguido de colocação do dreno de KEHR. Com o avanço tecnológico outras técnicas foram surgindo, sendo atualmente a mais utilizada a extração trans-duodenal através de papilotomia endoscópica (SAVASSI-ROCHA,1997). Por outro lado, quando o colédoco encontra-se muito dilatado, com atonia muscular, a cirurgia indicada deve ser a derivação bílio-digestiva (LYGIDADIS, 1984; ESCUDERO-FABRE et al.1991).

Apesar desses avanços, inúmeros estudos apontam que a morbimortalidade relacionada à coledocolitíase continua elevada (CSENDES et al., 1998, LEZOCHE; PAGANNI, 1996, SCHWESINGER, 1997).

Outros estudos de seguimento prolongado de portadores de litíase vesicular e coledocolitíase, incluindo pacientes com calculose residual após colecistectomia, confirmam a ocorrência elevada e progressiva de complicações, como icterícia obstrutiva, colangite e pancreatite aguda 
(COMFORT et al., 1948, HICKEN; McALLISTER, 1964, JOHNSON; HOSKING, 1987, LUND, 1960, PERISSAT et al., 1994, SCHWENSINGER, 1997 STRASBERG et al., 1996, WENCKERT; ROBERTSON, 1966; KOZOLL et al 1959).

A cirurgia por laparotomia para acesso à via biliar principal continua sendo o padrão referencial para o tratamento da coledocolitíase, procedendo-se a coledocolitotomia, retirada do cálculo e drenagem biliar externa. A palpação da via biliar, associada ou não à manobra de Kocher, consiste em importante etapa nesta avaliação intra-operatória (MADDEN, 1973, WAY, 1972).

$\mathrm{Na}$ década de 70, a introdução da colangiografia endoscópica retrógrada para o diagnóstico e subseqüente 0 surgimento da papilotomia(CLASSEN; SAFRANY, 1975, KAWAI, et al., 1974), permitiu uma alternativa terapêutica, principalmente naqueles pacientes já submetidos previamente à colecistectomia (COTTON et al., 1991, DANILEWITZ, 1989, LORIMER et al., 1997, MILLER at al., 1988, SIEGEL, 1981).

A introdução de procedimentos endoscópicos no armamento terapêutico da litíase na via biliar principal, a principio provocou resistência na comunidade cirúrgica, mas seu papel foi sendo estabelecido à medida que foi ocorrendo aperfeiçoamento para o manuseio do equipamento e redução das taxas de morbi-mortalidade (COTTON, 1984).

Cerca de 30 a $50 \%$ dos casos de litíase na via biliar principal apresentam-se de modo insuspeito, devido à baixa capacidade indicativa dos exames utilizados comumente na avaliação pré-operatória dos pacientes 
programados para colecistectomia (BERCI; MORGENSTEN, 1994, BERTHOU et al., 1998, KULLMAN et. Al., 1996, LEZOCHE, PAGANINI, 1995, MOREUX, 1995. PERISSAT et al., 1994, SCHWESINGER, 1997), reforçando-se o papel da colangiografia intra-operatória rotineira como única maneira de se estabelecer o diagnóstico nestas circunstâncias (GAMARODRIGUES, BRESCIANI, 1997, PINOTTI et al., 1999).

GIGOT et al. (1997) e PERISSAT et al. (1994), traçando um panorama acerca do tratamento da coledocolitíase na era pré-laparoscópica, reforçam o papel da colecistectomia e coledocolitotomia e salientam a importância do procedimento endoscópio nos casos de coledocolitíase complicada e em pacientes idosos e de alto risco cirúrgico (BERTHOU et al., 1998, LAl et al., 1992, NEOPTOLEMOS et al., 1987, STIEGMANN et al., 1992).

Com o advento da cirurgia videolaparoscópica, a abordagem endoscópica tornou-se um importante procedimento complementar, indicado em todas as fases do procedimento diagnóstico e terapêutico (préoperatório, trans-operatório e pós-operatório) (BOULAY et al., 1992, RANCESCHI et al., 1993, FRAZEE et al., 1993, KULLMAN et al., 1996, NEOPTOLEMOS et al., 1992, NEUHAUS et al., 1992, RIEGER, WAYANDO, 1995, SURICK et al., 1993).

À medida que a experiência com a via laparoscópica e os equipamentos foram se desenvolvendo, permitiu-se a coledocoscopia com aparelho flexível, sendo agora a exploração cirúrgica das vias biliares por essa via, uma realidade. 
BERCI (1993) afirma que o grande mérito da laparoscopia no tratamento da coledocolitíase é a sua resolução em um único tempo e quando esta possibilidade tornar-se largamente difundida, os procedimentos endoscópicos sobre a via biliar retomarão seu papel bem estabelecido, ou seja, nos pacientes de alto risco, na icterícia e colangite aguda, na calculose residual e nas complicações biliares, como descreveu FLETCHER (1994), afirmando que os princípios da cirurgia biliar não se modificaram, somente as técnicas do tratamento cirúrgico.

\section{2- Objetivos}

Dado a coledocolitíase residual apresentar grande complexidade no estabelecimento do seu diagnóstico e da proposta terapêutica temos como objetivo:

I - Avaliação de critérios clínicos, laboratoriais e métodos de imagem para diagnóstico.

II- Avaliar o resultado do tratamento da coledocolitíase residual através de procedimentos endoscópicos e cirúrgicos.

III- Avaliar a ocorrência de complicações e sua repercussão no período de internação hospitalar. 
2. CASUÍSTICA 


\section{2- CASUÍSTICA}

Foram estudados de forma retrospectiva, 32 (trinta e dois) pacientes portadores de coledocolitíase residual, internados na Clínica Cirúrgica do Hospital das Clínicas da Faculdade de Medicina da Universidade Federal de Goiás, no período de janeiro de 1995 a julho de 2001(Anexo-01).

Foram considerados como critérios de inclusão no presente estudo, os casos de coledocolitíase pós-colecistectomia, com informações nos prontuários que permitiram a análise das questões colocadas como objetivos do trabalho. Definiu-se como coledocolitíase residual a presença de cálculo(s) na via biliar principal pós-colecistectomia, incluindo-se os casos em que não se estabeleceu o diagnóstico no momento da colecistectomia (pela não realização da colangiografia trans-operatória ou por falha na sua interpretação) e aqueles em que não se decidiu pelo tratamento concomitante (coledocolitíase diagnosticada e não tratada), seja pela própria opção do cirurgião ou pelas condições clínicas do paciente.

A média etária foi 53,6 anos, variando entre 19 a 78 anos, sendo 11 do sexo masculino e 21 feminino correspondendo a $34,38 \%$ e $65,62 \%$ respectivamente. 
O estudo foi aprovado na Comissão de Ética para Análise de Projetos de Pesquisa - CAPPesg da Diretoria Clínica do Hospital das Clínicas da Faculdade de Medicina da Universidade de São Paulo sob o número 993/02. Aprovado em sessão de 11/12/2002. 
3. MÉTODOS 


\section{3- MÉTODOS}

\section{1- Avaliação diagnóstica de coledocolitíase residual}

O diagnóstico da coledocolitíase residual pode ser estabelecido através de critérios clínicos, laboratoriais e métodos de imagem.

\subsection{1- Critérios clínicos}

Os antecedentes valorizados como indicativos de coledocolitíase residual na história clínica, foram: icterícia, acompanhada de dor no andar superior do abdômen, com ou sem irradiação dorsal, ou crise sugestiva de pancreatite aguda.

\subsection{2- Análise laboratorial}

Os exames laboratoriais considerados pertinentes foram à dosagem plasmática da fração direta das bilirrubinas e de enzimas canaliculares hepáticas (fosfatase alcalina, Gama-Glutamiltransferase). 
Os exames foram considerados alterados quando acima do limite superior da normalidade, admitindo-se, para efeito de indicação de coledocolitíase a alteração de pelo menos um deles.

\subsection{3- Métodos de imagem}

\subsubsection{1- Ultra-sonografia}

Os informes ultra-sonográficos (FIGURA 1) estabelecidos foram a dilatação da via biliar, independente do segmento anatômico da via biliar principal avaliada, ou a evidência de cálculo intra-coledociano, produzindo sombra acústica posterior.
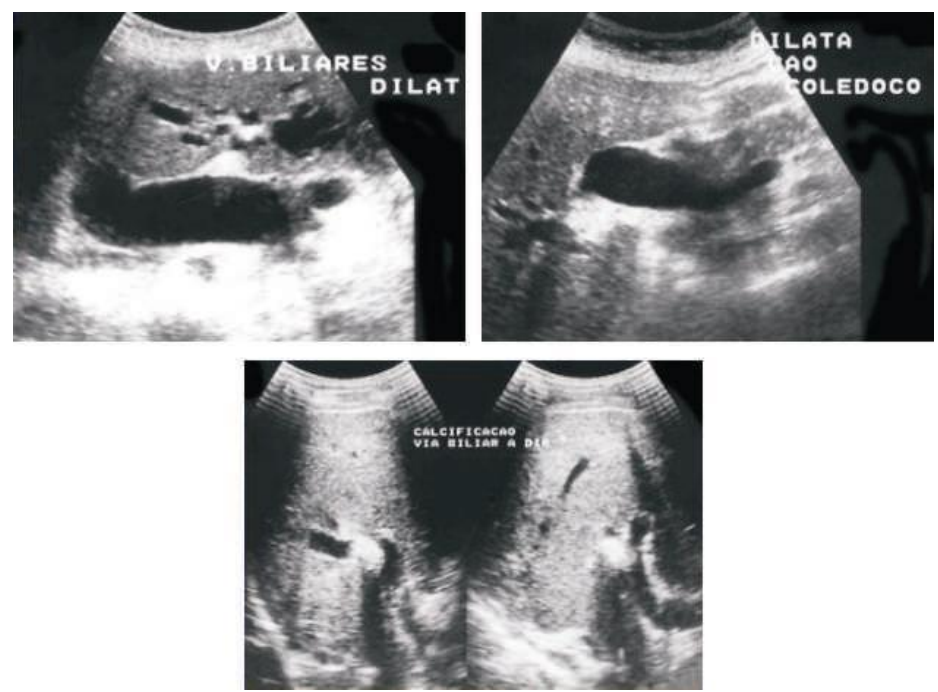

Figura 01-Ultra-sonografia nos dois primeiros diapositivos mostra apenas dilatação coledociana e na foto inferior a presença de cálculo no seu interior com a presença de dilatação de hepato-colédoco.

O exame ultra-sonográfico foi realizado em todos os pacientes, optando-se para a confirmação diagnóstica a realização de um dos métodos de imagem seguintes: 


\subsubsection{2- Colangiografia pelo dreno de Kehr}

Foi realizada naqueles pacientes previamente portadores de dreno de Kehr (FIGURA 2), introduzido por ocasião da colecistectomia.
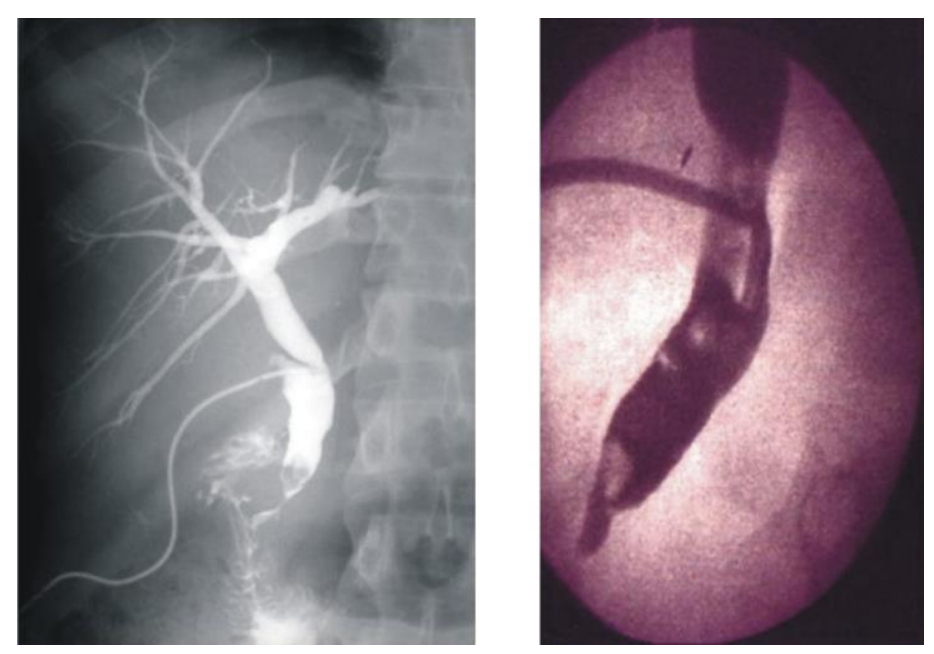

Figura 2- Colangiografia pelo dreno de Kehr onde podemos ver a presença de cálculo no interior da via biliar dilatada.

Para tal foi utilizado substancia de contraste hidrossolúvel a $30 \%$, através de infusão lenta pelo dreno, obtendo-se três radiografias (uma após infusão de 3 a $5 \mathrm{ml}$ de contraste, a segunda após três minutos, a terceira com infusão de 12 a $15 \mathrm{ml}$ de contraste).

\subsubsection{3- Colangio-pancreatografia retrógrada}

Método de confirmação diagnóstica de cálculo em via biliar, através de duodenoscopia (FIGURA 3) de visão lateral e cateterização da papila e injeção de contraste hidrossolúvel. Pode-se realizar o tratamento definitivo com papilotomia e extração do cálculo através de cateteres com balão ou cesta. 

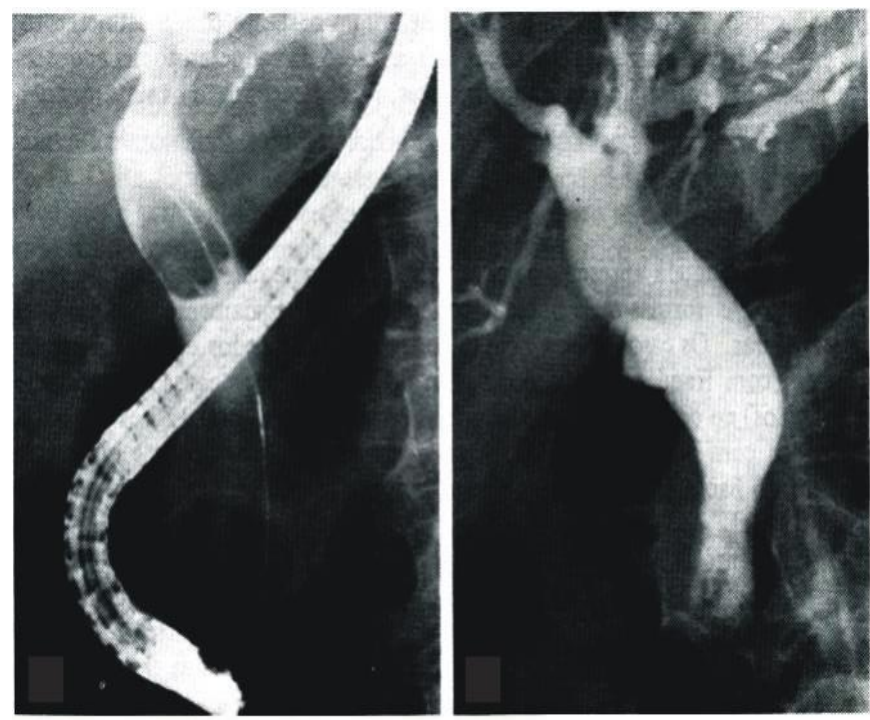

Figura 3 - Colangiopancreatografia retrógrada onde podemos ver a presença de colédoco dilatado com cálculo no seu interior e também impactado em papila duodenal.

\subsubsection{4- Ecoendoscopia}

Método complementar de diagnóstico de coledocolitíase que utiliza transdutor sonográfico acoplado na ponta do duodenoscópio (FIGURA 4). No presente estudo utilizamos o aparelho de ecoendoscopia da marca Olympus de alta freqüência.

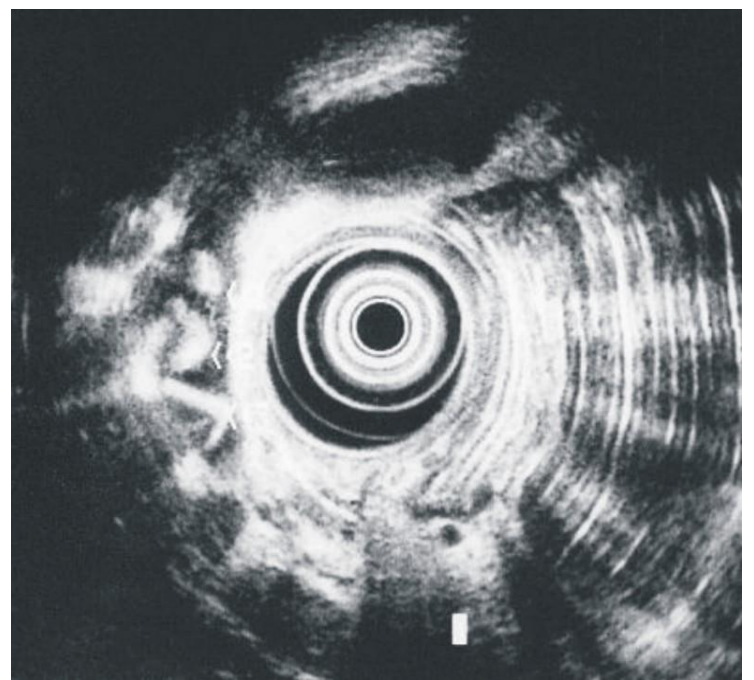

Figura 4 - Ecoendoscopia onde mostra a presença de cálculo no interior da via biliar principal dilatada. 


\subsubsection{5- Colangio-ressonância magnética}

A colangio-ressonância magnética (FIGURA 5) pode firmar o diagnóstico sem o uso de contraste na via biliar ou em corrente sangüínea, usando como meio de contraste a própria bile (por diferença de densidade). Identificam-se os segmentos da árvore biliar intra e extra-hepática e eventuais falhas de enchimento compatíveis com cálculos.

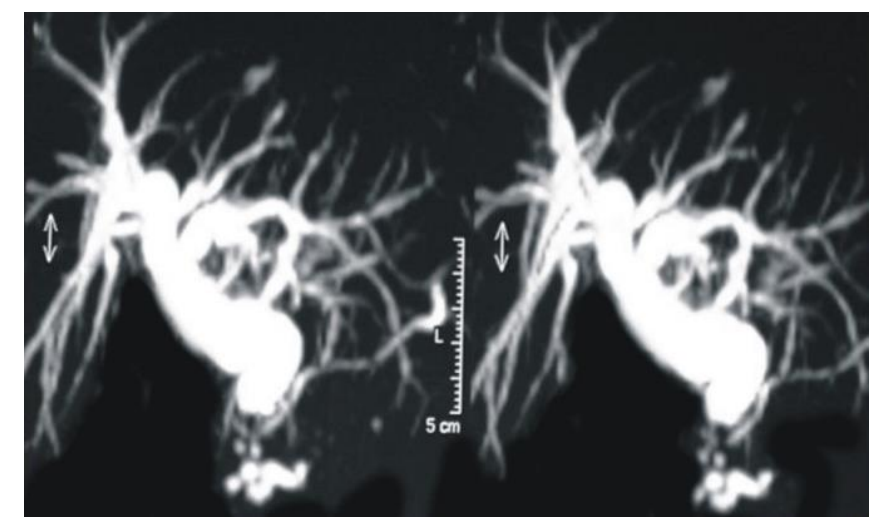

Figura 5 - Colangio-ressonância magnética onde mostra a presença de cálculo impactado em colédoco terminal com dilatação de via biliar principal.

\section{2- Procedimento terapêutico utilizado}

\subsection{1- Aspectos gerais}

Todos os pacientes haviam sido submetidos previamente à colecistectomia e após o diagnóstico da coledocolitíase foram encaminhados para o tratamento definitivo, endoscópico ou cirúrgico. 


\subsection{2- Papilotomia endoscópica}

Os pacientes receberam anestesia geral com entubação oro-traqueal e colocados em decúbito ventral laterizado para a esquerda com cabeça para a direita, presença de bocal de proteção da arcada dentária e introduzido o aparelho, passado o duodenoscópio até a segunda porção do duodeno, onde é localizada a papila que é identificada e cateterizada. A seguir é realizada papilotomia com papilótomo conectado à etetrocauterização e o clareamento da via biliar realizado com o uso de cateteres com balão e/ou cesta (FIGURA 6).
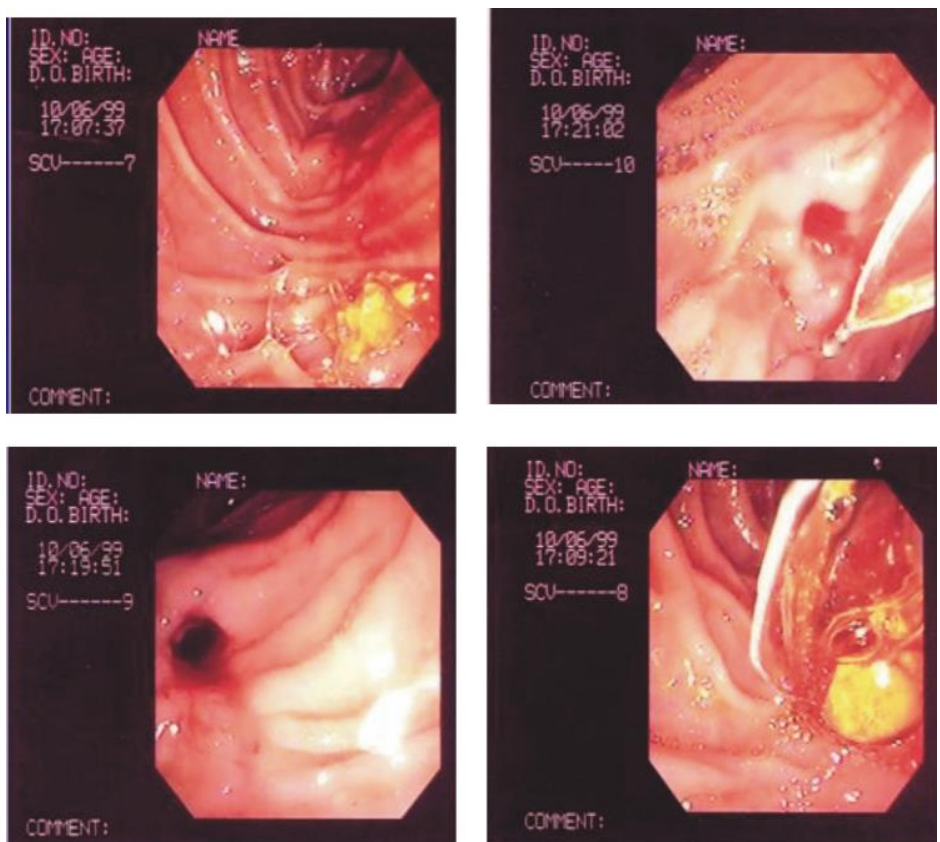

Figura 6 - Papilotomia onde podemos ver a papila já aberta com clareamento da via biliar principal com "Basket" e cálculo na luz intestinal. 


\subsection{3- Procedimento cirúrgico}

Os pacientes encaminhados para o procedimento cirúrgico receberam anestesia geral, sendo posicionados em decúbito dorsal horizontal com os braços abduzidos com ângulo de noventa graus.

As incisões cirúrgicas realizadas foram: mediana ou subcostal direita, de acordo com a cicatriz pré-existente. Abertura por planos com a realização cuidadosa da hemostasia, até a identificação do colédoco.

\subsubsection{1- Coledocolitomia com papilotomia trans-duodenal}

Após a individualização do colédoco, abertura do mesmo, na face anterior, e cateterização para identificar o local da papila no duodeno. Neste local, na face antero-lateral, realiza-se duodenotomia transversal, identificação da papila e, a seguir, papilotomia em posição de "onze" horas, retirados os cálculos tanto por via retrógrada como anterógrada (FIGURA 7). Completa-se suturando a mucosa do colédoco à mucosa duodenal, utilizando pontos simples com fio absorvível, realizando duodenorrafia com pontos simples de fio inabsorvível e rafia do colédoco com pontos simples de fio absorvível, observando-se o posicionamento do dreno de Kehr. Rotineiramente realiza-se colangiografia intra-operatória para o controle da posição do dreno e avaliação da via biliar. 


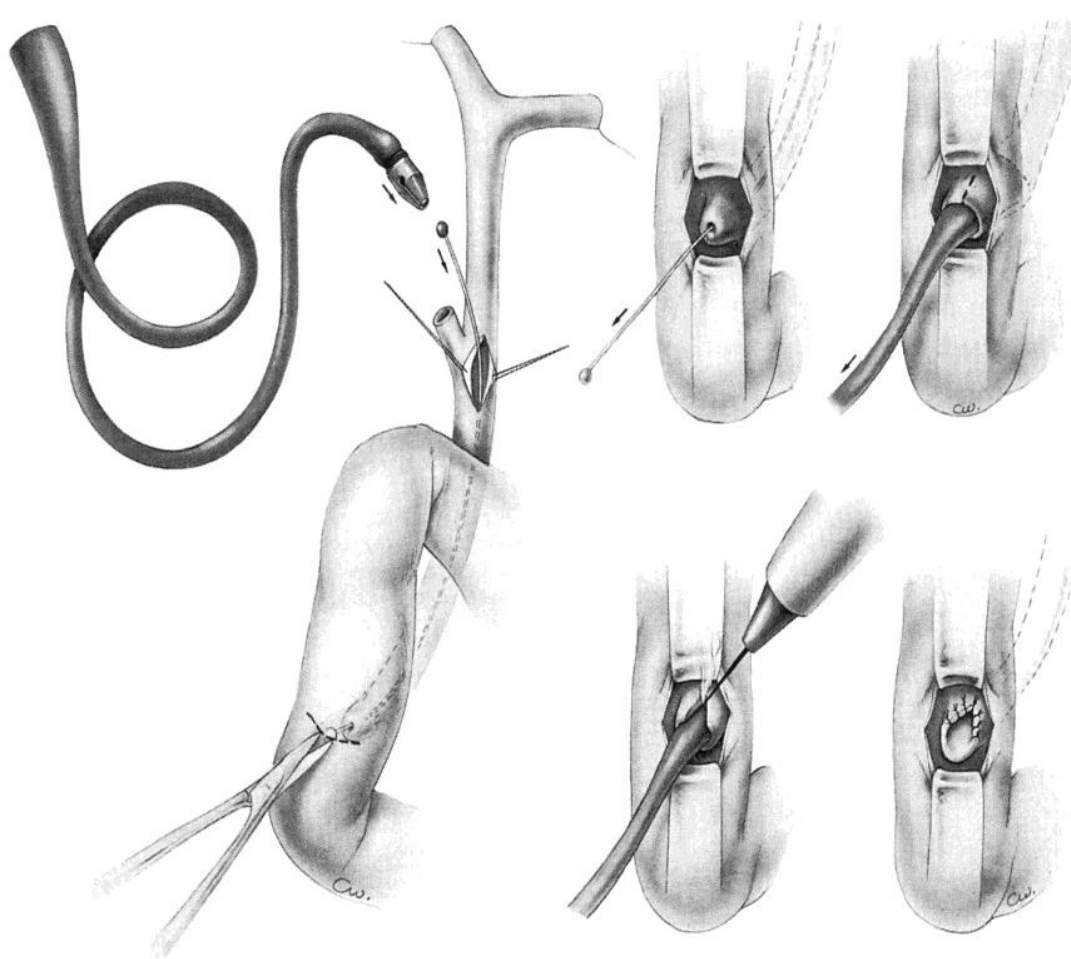

Figura 7 - Papilotomia trans-duodenal onde podemos ver a abertura do duodeno como identificar a papila com a realização da papilotomia.

\subsubsection{2- Derivação bílio-digestiva em Y-de-Roux}

Individualização do colédoco com secção, retirada dos cálculos e ligadura distal. Prepara-se uma alça de jejuno para a confecção do Y-deRoux (FIGURA 8), que pode ser realizada tanto pré-cólica como transmesocólica, com alça de jejuno de aproximadamente $40 \mathrm{~cm}$. 

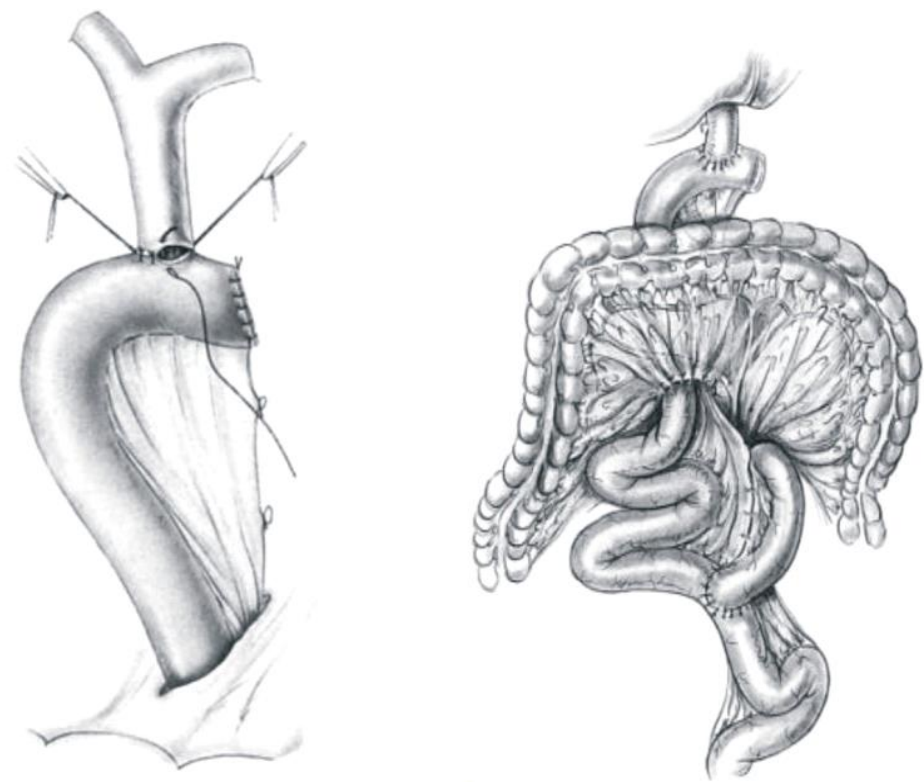

Figura 8 - Derivação em Y-de-Roux onde mostra a confecção trans-mesocólica.

\subsubsection{3- Derivação colédoco-duodenal}

Individualização do colédoco no 1/3 distal justa duodenal. Abertura transversal com a retirada dos cálculos do seu interior, duodenotomia e realizada anastomose colédoco-duodenal em plano único com fio absorvível e com pontos simples(FIGURA 9).
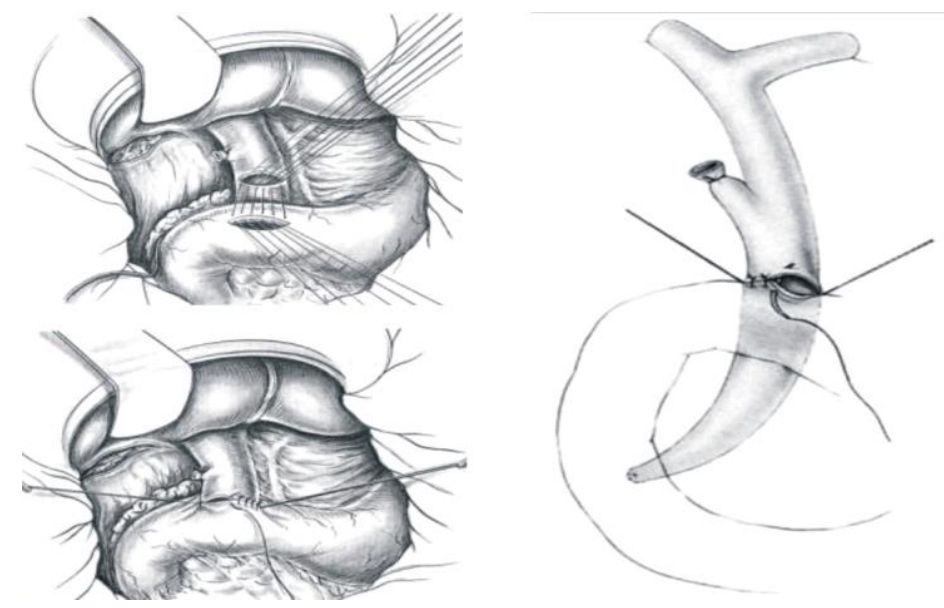

Figura 9 - Derivação colédoco-duodenal onde mostra a sua confecção laterolateral em colédoco dilatado. 


\section{3- Sucesso e insucesso no clareamento}

O sucesso no clareamento consiste na inexistência de qualquer cálculo remanescente na via biliar principal. Esta convicção sempre esteve amparada na realização de colangiografia trans-operatória, ou estudo dinâmico por fluoroscopia durante o procedimento endoscópico.

Os resultados foram submetidos à análise estatística.

\section{4- Complicações}

\subsection{1- Complicações endoscópicas}

As complicações relacionadas ao tratamento endoscópico podem ser precoces ou tardias. Aquelas são em decorrência direta da manipulação instrumental ao nível da papila duodenal e intra-coledociana, manifestandose imediatamente ou nas próximas horas após o exame, como: sangramento pela papila ou ductal, perfuração parietal da via biliar, pancreatite aguda e colangite; as tardias são relacionadas à evolução cicatricial local, determinando estenose a esse nível; para efeito deste estudo consideraram-se apenas os casos de pancreatite aguda com hiperamilasemia acompanhados de manifestações clinicas. 


\subsection{2- Complicações cirúrgicas}

As complicações cirúrgicas são as que ocorrem freqüentemente em todos os procedimentos realizados como: sangramentos, infecções e fístulas. Dependendo do procedimento utilizado, como a papilotomia, podem ocorrer pancreatite aguda com os mais variados graus de gravidade e tardiamente as estenoses.

\section{5- Período de internação pós-operatório}

A evolução clínica satisfatória foi o critério de definição para o momento da alta hospitalar, fundada principalmente no controle adequado da dor e capacidade de re-alimentação, assim como níveis normais da amilasemia, analisado no primeiro dia de pós-papilotomia e caso necessário nos subseqüentes.

Quando realizada drenagem da cavidade abdominal com dreno de Penrose, este foi mobilizado e retirado na evolução até a alta, desde que, inexistentes sinais indicativos de fístula biliar. Uma vez existindo drenagem biliar externa, a alta foi consumada normalmente após a colangiografia de controle, por volta do sétimo dia de pós-operatório; excepcionalmente estabeleceu-se a alta com o dreno biliar aberto, porém mantida a mesma programação de colangiografia de controle e fechamento do dreno.

Os resultados foram submetidos à analise estatística. 
4. RESULTADOS 


\section{4- RESULTADOS}

\section{1- Avaliação diagnóstica de coledocolitíase residual}

\subsection{1- Critérios clínicos}

Os antecedentes clínicos (TABELA 1) foram avaliados em todos os pacientes e foi observada a presença de icterícia acompanhada de dor em andar superior do abdômen em 23 (vinte e três) pacientes, dentre os quais, 4 (quatro) apresentavam manifestações clínicas exacerbadas compatíveis com pancreatite aguda. Os demais, 9 (nove) pacientes, apresentavam-se assintomáticos.

TABELA 1 - Critérios clínicos

\begin{tabular}{llc}
\hline & $\mathrm{N}^{\circ}$ & $\%$ \\
\hline Icterícia & $23^{*}$ & 71,9 \\
Assintomático & 09 & 28,1 \\
\hline
\end{tabular}

* 4 pacientes com pancreatite aguda 


\subsection{2- Análises laboratoriais}

Foram realizados exames laboratoriais (TABELA 2) em todos os pacientes. Houve alteração das dosagens de bilirrubina direta em 23 pacientes, da fosfatase alcalina em 30 (trinta) pacientes e da Gamaglutamiltransferase em 31 (trinta um) pacientes.

TABELA 2 - Análises laboratoriais - exames alterados

\begin{tabular}{llc}
\hline & $\mathrm{N}^{\circ}$ & $\%$ \\
\hline Bilirrubina direta & 23 & 71,9 \\
Fosfatase Alcalina & 30 & 93,7 \\
Gama-Glutamiltransferase & 31 & 96,8 \\
\hline
\end{tabular}

\subsection{3- Métodos de imagem}

\subsubsection{1- Ultra-sonografia}

Todos os pacientes realizaram ultra-sonografia (TABELA 3), sendo que apenas 3 (três) casos mostravam a presença de cálculo no interior da via biliar principal; em 14 (quatorze) pacientes observava-se apenas dilatação sem evidenciar a causa da dilatação. Em 15 (quinze) pacientes o colédoco mostrava-se sem qualquer alteração. 
TABELA 3 - Ultra-sonografia

\begin{tabular}{lcc}
\hline & $N^{\circ}$ & $\%$ \\
\hline Dilatação & $17^{*}$ & 53,1 \\
Normal & 15 & 46,9 \\
\hline
\end{tabular}

* presença de cálculo em 3 casos $(9,4 \%)$

\subsubsection{2- Colangiografia pelo dreno Kehr}

Havia 3 (três) pacientes com dreno de Kehr, por isso a confirmação diagnóstica foi através da colangiografia pelo próprio dreno de Kehr. Houve confirmação do diagnóstico em todos os casos.

\subsubsection{3- Colangiopancreatografia retrógrada}

Foi realizada em 12 (doze) pacientes, com confirmação diagnóstica de coledocolitíase residual em todos eles.

\subsubsection{4- Ecoendoscopia}

Para a confirmação diagnóstica a ecoendoscopia foi realizada em 13 (treze) pacientes. Houve confirmação da presença de cálculos em todos os casos.

\subsubsection{5- Colangioressonância magnética}

A colangiografia por ressonância magnética foi realizada em 4 (quatro) pacientes, sendo o diagnóstico de coledocolitíase confirmado em todos eles. 


\section{2- Procedimentos utilizados}

\subsection{1- Papilotomia endoscópica}

O procedimento endoscópico, método utilizado com a finalidade de realizar a papilotomia e o clareamento (FIGURA 1) da via biliar, foi empregado em 27 pacientes já com diagnóstico definitivo. Em dois casos não foi possível a realização do clareamento, sendo os pacientes encaminhados para o procedimento cirúrgico.

\subsection{2- Procedimentos cirúrgicos}

O procedimento cirúrgico, por laparotomia, foi empregado em 7 (sete) pacientes.

\subsubsection{1- Coledocolitomia com papilotomia trans-duodenal}

A coledocolitomia com papilotomia trans-duodenal (FIGURA 2) foi realizada em 3 (três) pacientes.

\subsubsection{2- Derivação bílio-digestiva em Y-de-Roux}

Com a presença de dilatação importante e atonia do colédoco a derivação bílio-digestiva com a confecção de y-de-Roux (FIGURA 3) foi empregada em 3 (três) pacientes. 


\subsubsection{3- Derivação colédoco-duodenal}

Foi realizado um caso de derivação colédoco-duodenal (FIGURA 4), pós-extração do cálculo, devido ao paciente apresentar idade avançada e condições clínicas inadequadas no momento da intervenção, para um procedimento cirúrgico mais prolongado.

\section{3- Sucesso e insucesso no clareamento}

\subsection{1- Endoscópico}

O sucesso no clareamento da via biliar principal foi obtido em 25 (vinte e cinco) pacientes, correspondendo a 92,5\%. Dois pacientes, correspondendo a 7,5\%, nos quais não foi possível a extração do(s) cálculo(s) por via endoscópica, foram encaminhados ao procedimento cirúrgico.

\subsection{2- Cirúrgico}

Todos os 7(sete) pacientes submetidos a tratamento cirúrgico obtiveram sucesso no clareamento da via biliar. 
Não houve diferença significativa entre as taxas de sucesso obtidas com os procedimentos endoscópico e cirúrgico (TABELA 4) realizados (Teste Exato de Fisher).

TABELA 4 - Sucesso no clareamento

\begin{tabular}{lcc}
\hline & $N^{\circ}$ & $\%$ \\
\hline Endoscópico & 25 & 92,5 \\
Cirúrgico & 07 & 100 \\
\hline
\end{tabular}

$P=0,626$

\section{4- Complicações}

Não houve mortalidade em nenhum dos pacientes.

Dentre os pacientes submetidos a tratamento cirúrgico não houve complicação intra-abdominal. Apenas um paciente apresentou hematoma de parede abdominal que motivou re-operação para a limpeza da ferida operatória.

Dentre aqueles submetidos a tratamento endoscópico houve 2 (dois) casos de pancreatite aguda, mantidos sob tratamento clínico e de evolução favorável. Houve um caso de hemorragia ao nível da papila duodenal que necessitou transfusão de $600 \mathrm{ml}$ de concentrado de hemácias; na revisão endoscópica o sangramento já havia cessado. 
Não houve diferença significativa entre as taxas de complicações dos procedimentos cirúrgico e endoscópico (Teste Exato de Fisher) (TABELA 5).

TABELA 5 - Complicações

\begin{tabular}{lll}
\hline & $\mathrm{N}^{\circ}$ & $\%$ \\
\hline Cirúrgico & 01 & 14,3 \\
Endoscópico & & \\
Pancreatite & 02 & 08 \\
Hemorragia & 01 & 04 \\
\hline $\mathrm{P}=0,448$ & &
\end{tabular}

\section{5- Período de internação}

O período de internação variou com o procedimento realizado, sendo que, dentre os pacientes submetidos a tratamento endoscópico, 20 (vinte) deles, correspondendo a $80 \%$, tiveram alta com dois dias. Apenas um caso teve internação por onze dias devido ao quadro de pancreatite.

Dentre os pacientes submetidos a procedimentos cirúrgicos 6(seis) deles correspondendo a $86 \%$ tiveram alta entre o $3^{\circ}$ e $7^{\circ}$ dia de pós-operatório, e apenas um paciente teve internação prolongada (9 dias) devido à necessidade de re-operação para limpeza de hematoma da parede abdominal. 
Houve diferença estatística significativa entre o período de internação dos pacientes nos procedimentos endoscópicos e cirúrgicos (Teste de kolmogorov-smirnov) (TABELA 6).

TABELA 6 - Período de internação em dias pós-procedimento

\begin{tabular}{lcccc}
\hline & \multicolumn{2}{c}{ Cirúrgico(N=7) } & \multicolumn{2}{c}{ Endoscópico(N=25) } \\
\hline & $\mathrm{N}^{\circ}$ & $\%$ & $\mathrm{~N}^{\circ}$ & $\%$ \\
\hline $0-2$ & 0 & 0 & 20 & 80 \\
$3-7$ & 06 & 86 & 04 & 16 \\
$>7$ & 01 & 14 & 01 & 04 \\
\hline
\end{tabular}

$Z=1,871 ; p=0,0002$ (teste kolmogorov-smirnov) 
5. DISCUSSÃO 


\section{5- DISCUSSÃO}

$\mathrm{Na}$ clínica cirúrgica da Faculdade de Medicina da UFG as colangiografias trans-operatórias são realizadas de rotina nas colecistectomias, dadas as imensas vantagens que o método propicia (LIBANORI et al., 1995, PINOTTI et al.,1999, GAMA-RODRIGRES et al., 1996) em especial no que se refere ao diagnóstico de coledocolitíase cuja prevalência varia de 3\% a 9\% (BERCI G, MORGENSTERN, 1994; SAVASSIROCHA et al. 1997, SANTO., 2000). Em serviços onde ocorre maior contingente de pacientes idosos e com colecistite aguda, observa-se incidência que varia de 18\% a 35\% (HUGUIER et al., 1991, PHILLIPS, 1994, ROSENTAL et al., 1998).

GIGOT et al. (1997) relatam experiência acumulada ao longo de 2500 colecistectomias e realizando a colangiografia intra-operatória diagnosticaram 90 casos de coledocolitíase, perfazendo 3,6\% da casuística.

No período compreendido de janeiro de 1995 a julho de 2001 foram realizados, na clínica cirúrgica da Faculdade de Medicina da UFG, 882 procedimentos biliares, englobando colecistectomia e operações diretamente 
sobre o colédoco. Cento e vinte e cinco pacientes eram portadores de coledocolitíase, dos quais 32 na forma residual (ANEXO 2).

A litíase biliar tem nítido predomínio no sexo feminino, mantendo esta relação quando do comprometimento da via biliar principal (MOREAUX, 1995, WAY et al., 1972).

Em nossa casuística 21(vinte um) do sexo feminino, sendo 65,62\% e 11 (onze) do sexo masculino, sendo $34,38 \%$, e a idade média de 53,6 anos.

\section{1- Avaliação diagnóstica da coledocolitíase residual}

\subsection{1- Critérios clínicos}

Antecedentes clínicos de icterícia e pancreatite aguda são indicativos de coledocolitíase, mas sua ausência não afasta esta possibilidade; pelo contrário, um grande contingente dos pacientes portadores de litíase na via biliar principal não refere estes antecedentes (BERCl; MORGENSTERN, 1994). Há de considerar-se também que a presença de icterícia na vigência de colelitíase não implica na presença de cálculos na via biliar principal (WAY et al.,1972).

$\mathrm{Na}$ nossa análise a icterícia esteve presente em 23 (vinte três) dos pacientes, dentre os quais a pancreatite aguda associada em 4(quatro) casos. Nove pacientes, correspondendo a $28,1 \%$ apresentavam-se assintomáticos. 


\subsection{2- Análises laboratoriais}

No que tange aos exames laboratoriais para efeito de indicação de coledocolitíase, alterações plasmáticas de pelo menos uma das enzimas hepáticas ou da fração direta das bilirrubinas têm sido valorizadas, em especial a dosagem plasmática da fosfatase alcalina, quando o resultado está duas vezes acima do valor normal (STAIN et al., 1994, BARKUN et al., 1994, SANTUCCI et al., 1996).

No entanto BERCI E MORGENSTERN (1994) ressaltaram a baixa sensibilidade dos exames laboratoriais como indicativos de codocolitíase, não se afastando essa possibilidade quando normais.

$\mathrm{Na}$ nossa casuística as alterações laboratoriais referentes à dosagem plasmática da fração direta de bilirrubina, fosfatase alcalina e Gamaglutamiltransferase estiveram presentes em mais de $70 \%$ dos casos. Provavelmente pelo fato de se tratarem de pacientes com coledocolitíase residual, portanto, história clínica mais prolongada, com maior repercussão sobre a via biliar.

\subsection{3- Métodos de imagem}

\subsubsection{1- Ultra-sonografia}

As limitações do ultra-som para a avaliação da via biliar extra-hepática com vista à detecção de coledocolitíase, reproduz-se em vários estudos na literatura (CRONAN,1986, HUNTER; SOPER, 1992, MONTEIRO DA CUNHA et al.,1994, STRASBERG et al.,1996). 
$\mathrm{Na}$ nossa casuística a presença de cálculo na via biliar principal foi de $9,4 \%$, sendo que em $45,7 \%$ dos casos a ultra-sonografia mostrou apenas dilatação sem a causa desta; em 46,9\% dos pacientes o exame mostrou-se normal.

A sensibilidade global do ultra-som na detecção de coledocolitíase varia aproximadamente de $19 \%$ a $55 \%$, aumentando nos casos de icterícia associada(BERTHOU et al.,1998, CRONAN,1986, JOYCE et al.,1991,PERISSAT et al.,1994, SURIK et al.,1993, SCHWESINGER, 1997). Numa série de 121 portadores de coledolitíase, MILLAT et al (1995) estabeleceram o diagnóstico de coledocolitíase em apenas 34\% deles. BERCI e MORGENSTERN (1994) demonstraram que $27 \%$ dos pacientes com coledocolitíase apresentavam algum grau de dilatação biliar ao exame ultra-sonográfico, evidenciando cálculo na luz biliar em apenas 13\%. Ou seja, em $60 \%$ dos pacientes a ultra-sonografia foi incapaz de sugerir o diagnóstico de coledocolitíase.

\subsubsection{2- Outros Métodos de Imagem}

\subsubsection{2a- Colangiografia pelo Kehr}

Houve 3 (três) casos onde os pacientes deram entrada no serviço com dreno de Kehr na via biliar e com isso a confirmação diagnóstica foi facilitada pela realização de colangiografia. O diagnóstico foi confirmado em todos os casos. 


\subsubsection{2b- Colangiopancreatografia retrógrada}

Exame de alta definição e elevada acurácia, representa o padrão referencial de diagnóstico da coledocolitíase.

Quando realizada no período pós-operatório e dispondo da presença de dreno biliar externo, propiciando a passagem de fio guia, o sucesso na cateterização da papila duodenal é ainda mais tangível (BERCl; MORGENSTERN, 1994; HUNTER; SOPER, 1992, FITZGIBBONS et al., 1995).

Tendo sido realizada em 12 (doze) pacientes, o diagnóstico foi confirmado em todos os casos.

\subsubsection{2c- Ecoendoscopia}

A ecoendoscopia também representa exame de alta definição e elevada acurácia. Permite estabelecer o diagnóstico de coledocolitíase mesmo em ductos não dilatados(AMOUYAL et al., 1994, D'IPPOLITO et al.,1998, EDMUNDOWICZ et al.,1994,SALMERON et al.,1994).

No presente estudo a ecoendoscopia foi realizada em 12(doze) pacientes e o diagnóstico confirmado em todos os casos.

\subsubsection{2d- Colangiorressonância magnética}

A colangiografia por ressonância magnética também consiste em exame de alta precisão. Tal como a ecoendoscopia, é um exame de custo elevado, o que inviabiliza sua utilização corriqueira(BERTHOU et al. 1998, PAVONE et al.,1997, ROSENTAL et al.,1998).

$\mathrm{Na}$ nossa casuística a colangiorressônancia foi realizada em 04(quatro) pacientes e em todos foi confirmado o diagnóstico. 


\section{2- Procedimentos utilizados}

A complexidade do enfoque terapêutico da coledocolitíase é evidenciada pela multiplicidade de proposições técnicas(SANTO,2000).

Em 1993, reunião de consenso promovida pelo Instituto Nacional de Saúde dos EUA subscrevia todos as formas bem estabelecidas de tratamento da litíase na via biliar principal: laparotomia, laparoscopia e endoscopia, incluindo a conduta expectante em situações especiais, destacando o papel e perspectiva que a via laparoscópica vinha adquirindo(NIH CONSENSUS,1993, HARDY,1994). Estudo multi-cêntrico coordenado por SAVASSI-ROCHA et al (1997) demonstra essa complexidade, no qual a abordagem endoscópica representou 55\% dos casos tratados de coledocolitíase.

STRASBERG et al (1996) sumarizando casuísticas de terapêutica endoscópica(ALIPERTI et al., 1991, GRAHAM et al., 1993, BARKUN et al., 1993, FRAZEE et al., 1993, RIEGER et al 1994, SALKY; BAUER, 1994), demonstram índices de sucesso variando de 90 a 100\%.

$\mathrm{Na}$ nossa casuística não observamos diferença significativa entre as taxas de sucesso obtidas com o tratamento endoscópico e cirúrgico(92,5\% e $100 \%$ respectivamente). 


\subsection{1- Procedimento endoscópico}

A experiência adquirida nos últimos anos permitiu ao procedimento endoscópico atingir elevados ìndices de sucesso no tratamento das afecções biliares(DAVIS et al., 1997).

No entanto, a taxa de sucesso com este procedimento tem sido descrita em torno de 90\% (COPPOLA et al., COTTON et al., 1991, NEOPTOLEMOS, 1987,NEUHAUS et al., 1989). COTTON (1984) e LAMBERT et al. (1991) destacam que os índices de sucesso devem incluir tanto os procedimentos de esfincterotomia quanto o da extração.

PERISSAT et al. (1994) e SAKAI et al. (1999) referem que o procedimento endoscópico, utilizando técnicas básicas com sondas tipo "cesta" e cateter balonado, atinge índices de sucesso em torno de $90 \% . \mathrm{Na}$ impossibilidade de remoção endoscópica do(s) cálculo(s), principalmente nos cálculos grandes, pode-se empregar uma endoprotese biliar para evitarse a impactação e manter o ducto pérvio até a solução definitiva (SAKAl et al., 1999).

Em nossa casuística 27 (vinte sete) pacientes foram encaminhados ao procedimento endoscópico, sendo que o sucesso foi alcançado em 25 (vinte e cinco) deles, e o insucesso, em 2 (dois) casos, que foram encaminhados para o procedimento cirúrgico. 


\subsection{2- Procedimento cirúrgico}

A indicação para derivação bilio-digestiva é favorecida quando estiver presente grave comprometimento coledociano, evidenciado por grande dilatação biliar e acentuada dificuldade no esvaziamento trans-papilar com atonia coledociana evidenciada a colangiografia intra-operatória, principalmente se associado à calculose múltipla(ESCUDERO-FEBRE et al., 1991, LYGIDAKIS, 1984). Não se deve, contudo, indicá-lo exageradamente, uma vez que a intensidade do processo inflamatório, preferencialmente crônico, presente na parede do colédoco, não está relacionada com seu grau de dilatação e, portanto, não constituem alterações irreversíveis (CECCONELO,1979).

Nos 3 (três) pacientes em que se realizou derivação bílio-digestiva todos eles se apresentavam com colédoco de calibre muito aumentado e atônico, sendo realizada a coledocotomia com clareamento da via biliar e confeccionado a derivação bílio-digestiva em Y-de-Roux.

Em 3 (três) pacientes, com colédoco sem grande dilatação, foram realizadas a coledocotomia e duodenotomia com identificação da papila e confecção da papilotomia. Realizado o clareamento da via biliar principal, deixou-se no colédoco um dreno de Kehr, com a finalidade de proteção da anastomose e prevenção de possível fístula biliar. A técnica da esfincteroplastia (papilotomia) foi bem ilustrada por JONES E SMITH (1972).

Em um paciente, devido às condições clínicas, foi realizada a coledocotomia anterior com clareamento da via biliar e realização de 
derivação colédoco-duodenal. Destaca-se a importância da realização de anastomose duodeno-jejunal com, no mínimo, 15mm de diâmetro, com o objetivo de garantir adequada permeabilidade e evitar complicações futuras como uma eventual estenose(ESCUDERO-FABRE 1991).

\section{3- Complicações}

Não houve nenhum óbito em nossa casuística. Ocorreram dois casos de pancreatite e um caso de hemorragia digestiva. Isso nos procedimentos endoscópicos, correspondendo a cerca de 11\%, sendo resolvidos com tratamento clínico.

Nos procedimentos cirúrgicos ocorreu apenas um caso de hematoma de parede abdominal com a necessidade de drenagem. Não houve qualquer outra complicação na casuística.

Após coledocotomia, BERTHOU et al. (1998), DION et al. (1994), MILLAT et al. (1995) e ROBINSON et al., (1995) apontam incidência aproximada de 3(três) a 7\% de complicações.

Reunião de consenso versando sobre complicações da papilotomia endoscópica sugere que o índice de morbidade gira em torno de $10 \%$ e de mortalidade por volta de 1,5\%, havendo necessidade de tratamento cirúrgico em menos de $20 \%$ das complicações. Uma das complicações endoscópicas mais temidas, que ocorre em cerca de $1 \%$ dos procedimentos, é a perfuração parietal (janela posterior), implicando altas taxas de intervenções 
cirúrgicas complementares e mortalidade de $25 \%$ e $15 \%$ respectivamente (COTTON et al., 1991).

Os vários relatos na literatura apontam para índices de morbidade variando aproximadamente de $3 \%$ a $15 \%$ e de mortalidade entre $0,3 \%$ e $3 \%$ (CHAMPAULT, 1993, COPALLA et al, 1997, JOHNSON et al., 1993, LAMBERT et al., 1991, LANDONI et al ., 1993, MILLER et al., 1988, NEUHAUS et al 1992).

\section{4- Período de internação pós-operatório}

O critério clínico para definição do momento da alta, atestado pela evolução pós-operatória favorável, controle da dor e adequada capacidade de realimentação, é universal, fundado na própria experiência da prática cirúrgica. Com a manipulação sobre o esfíncter de Oddi, comprometendo o esvaziamento bilio-pancreático, o controle da amilasemia pós-manipulação cirúrgica e endoscópica se faz necessário, como parâmetro para definição de alta.

$\mathrm{Na}$ nossa casuística, 20(vinte) pacientes, correspondendo a $80 \%$, tiveram alta no segundo dia pós-procedimento endoscópico, e 4 até o sétimo dia. Apenas um paciente teve internação mais prolongada, onze dias, correspondendo a $4 \%$.

Nos procedimentos cirúrgicos os pacientes tiveram alta apenas após o quarto dia de pós-operatório, sendo: 3 (três) pacientes, com alta no quarto dia, correspondendo a $42,86 \%$ dos casos cirúrgicos, mais 3 (três) pacientes 
com alta no quinto dia e um paciente correspondendo a $14,28 \%$ com alta no décimo dia.

Da análise estatística (teste de Klmogorov-Smirnov $z=1,871 ; p=0,002$ ) observa-se diferença significativa, quanto ao período de internação, entre os procedimentos utilizados, endoscópicos e cirúrgicos, evidenciando a necessidade da internação mais longa nos pacientes operados. Ressalte-se, contudo, que cerca de $85 \%$ destes pacientes tiveram alta até o 5ํㅜㄹ dia de pósoperatório, suficiente para resolução de afecção tão complexa como a coledocolitíase 
6. CONCLUSÃO 


\section{6- Conclusão}

- A maioria dos pacientes portadores de coledocolitíase residual teve como sintoma principal a icterícia.

- A ultra-sonografia não consistiu em método diagnóstico eficaz para a coledocolitíase residual.

- Tanto o procedimento endoscópico quanto o cirúrgico mostraram altos índices de sucesso no clareamento da via biliar.

- Em ambos os procedimentos, a morbidade foi baixa e a mortalidade nula.

- O período de internação foi menor quando o procedimento endoscópico foi realizado. 
7. ANEXOS

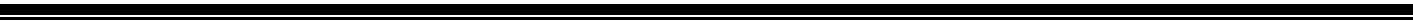




\section{7- Anexos}

ANEXO 1 - Identificação e relação de pacientes com cálculo residual

\begin{tabular}{|c|c|c|c|c|c|c|c|c|c|c|}
\hline Nome & PRON. & $\mathbf{N}^{\circ}$ & Sexo & Idade & Peso & Altura & Icterícia & BT & BD & BI \\
\hline MJC & 5001490 & 01 & $F$ & 53 & 65 & 1,57 & + & $A$ & $A$ & $\mathrm{~N}$ \\
\hline VAN & 4638706 & 02 & $\mathrm{~F}$ & 19 & 69 & 1,68 & $\mathrm{~N}$ & $\mathrm{~N}$ & $\mathrm{~N}$ & $\mathrm{~N}$ \\
\hline SRS & 4866604 & 03 & $F$ & 40 & 57 & 1,42 & + & $A$ & $A$ & $\mathrm{~N}$ \\
\hline JRF & 4250502 & 04 & $M$ & 51 & 70 & 1,65 & $\mathrm{~N}$ & $\mathrm{~N}$ & $\mathrm{~N}$ & $\mathrm{~N}$ \\
\hline RMDC & 2671386 & 05 & $\mathrm{~F}$ & 58 & 52 & 1,49 & + & A & A & $\mathrm{N}$ \\
\hline$P Q G$ & 492119 & 06 & $M$ & 78 & 54 & 1,56 & $\mathrm{~N}$ & $\mathrm{~N}$ & $\mathrm{~N}$ & $\mathrm{~N}$ \\
\hline LB & 4777876 & 07 & $M$ & 56 & 64 & 1,62 & + & $A$ & $A$ & $\mathrm{~N}$ \\
\hline MAS & 5181763 & 08 & $\mathrm{~F}$ & 39 & 57 & 1,56 & + & A & A & $\mathrm{N}$ \\
\hline JFB & 4766234 & 09 & $F$ & 54 & 67 & 1,62 & + & $A$ & $A$ & $\mathrm{~N}$ \\
\hline OBS & 3805 & 10 & $F$ & 50 & 68,5 & 1,70 & $\mathrm{~N}$ & $\mathrm{~N}$ & $\mathrm{~N}$ & $\mathrm{~N}$ \\
\hline APV & 7311599 & 11 & $\mathrm{~F}$ & 67 & 57 & 1,49 & + & $A$ & $A$ & $\mathrm{~N}$ \\
\hline SJL & 3735982 & 12 & $M$ & 61 & 54 & 1,49 & + & $A$ & $A$ & $\mathrm{~N}$ \\
\hline NAS & 3885597 & 13 & $M$ & 61 & 51 & 1,40 & $\mathrm{~N}$ & $\mathrm{~N}$ & $\mathrm{~N}$ & $\mathrm{~N}$ \\
\hline DSS & 2283015 & 14 & $\mathrm{~F}$ & 49 & 76 & 1,68 & +++ & $A$ & A & $A$ \\
\hline MCPS & 3392123 & 15 & $\mathrm{~F}$ & 53 & 57 & 1,65 & + & $A$ & $A$ & $\mathrm{~N}$ \\
\hline SSL & 4990909 & 16 & $F$ & 58 & 68 & 1,60 & + & $A$ & $A$ & $\mathrm{~N}$ \\
\hline MAR & 5152590 & 17 & $\mathrm{~F}$ & 48 & 59 & 1,57 & + & $A$ & $A$ & $A$ \\
\hline MRM & 3247475 & 18 & $M$ & 54 & 54 & 1,59 & $\mathrm{~N}$ & $\mathrm{~N}$ & $\mathrm{~N}$ & $A$ \\
\hline ZFA & 4963336 & 19 & $\mathrm{~F}$ & 42,2 & 63, & 1,58 & $\mathrm{~N}$ & $\mathrm{~N}$ & $\mathrm{~N}$ & $\mathrm{~N}$ \\
\hline VLA & 5172416 & 20 & $\mathrm{~F}$ & 62 & 78 & 1,65 & + & $A$ & $A$ & $\mathrm{~N}$ \\
\hline CAC & 2793669 & 21 & $\mathrm{~F}$ & 54 & 67 & 1,68 & + & $A$ & $A$ & $\mathrm{~N}$ \\
\hline MD & 496 & 22 & $M$ & 54 & 65 & 1,59 & + & $A$ & $A$ & $\mathrm{~N}$ \\
\hline SSG & 462 & 23 & $M$ & 61 & 68 & 1,58 & + & $A$ & A & $\mathrm{N}$ \\
\hline MPSR & 515252 & 24 & $\mathrm{~F}$ & 59 & 62 & 1,57 & $\mathrm{~N}$ & $\mathrm{~N}$ & $\mathrm{~N}$ & $\mathrm{~N}$ \\
\hline LMS & 5171848 & 25 & $\mathrm{~F}$ & 50 & 64 & 1,68 & + & $A$ & A & $\mathrm{N}$ \\
\hline NGM & 5289939 & 26 & $F$ & 56 & 58 & 1,57 & $\mathrm{~N}$ & $\mathrm{~N}$ & $\mathrm{~N}$ & $\mathrm{~N}$ \\
\hline SPL & 4415444 & 27 & M & 49 & 68 & 1,58 & + & A & $A$ & $\mathrm{~N}$ \\
\hline FSO & 5184502 & 28 & $M$ & 59 & 78 & 1,66 & + & A & A & $\mathrm{N}$ \\
\hline SRAM & 4998811 & 29 & $\mathrm{~F}$ & 63 & 69 & 1,65 & + & $A$ & A & $\mathrm{N}$ \\
\hline OMJ & 4662128 & 30 & $M$ & 60 & 69 & 1,68 & + & $A$ & $A$ & $\mathrm{~N}$ \\
\hline MFJ & 5001441 & 31 & $\mathrm{~F}$ & 53 & 61 & 1,59 & + & $A$ & $A$ & $\mathrm{~N}$ \\
\hline MFD & 4676094 & 32 & $\mathrm{~F}$ & 42 & 65 & 1,68 & + & A & A & $\mathrm{N}$ \\
\hline
\end{tabular}

PRON= Prontuário do paciente no hospital

$\mathrm{BT}=$ Bilirrubinas totais

$\mathrm{BD}=$ Bilirrubinas direta

$\mathrm{BI}=$ Bilirrubinas indireta 


\begin{tabular}{|c|c|c|c|c|c|c|c|c|c|c|c|}
\hline $\mathbf{N}^{\circ}$ & FA & GGT & AMIL & LIP & TGO & TGP & USG & C.K & CPRE & CR & ECC \\
\hline 01 & $A$ & $A$ & $\mathrm{~N}$ & $\mathrm{~N}$ & $\mathrm{~N}$ & $\mathrm{~N}$ & $\mathrm{~N}$ & $\mathrm{NR}$ & NR & NR & $R$ \\
\hline 02 & $\mathrm{~N}$ & $A$ & $\mathrm{~N}$ & $\mathrm{~N}$ & $\mathrm{~N}$ & $\mathrm{~N}$ & $\mathrm{~N}$ & $\mathrm{R}$ & NR & NR & $\mathrm{NR}$ \\
\hline 03 & $A$ & $A$ & $\mathrm{~N}$ & $\mathrm{~N}$ & $\mathrm{~N}$ & $\mathrm{~N}$ & $\mathrm{D}$ & NR & NR & $\mathrm{R}$ & $\mathrm{NR}$ \\
\hline 04 & $A$ & $A$ & $\mathrm{~N}$ & $\mathrm{~N}$ & $\mathrm{~N}$ & $\mathrm{~N}$ & $\mathrm{DC}$ & $\mathrm{NR}$ & $\mathrm{R}$ & NR & $\mathrm{NR}$ \\
\hline 05 & $A$ & $A$ & $A$ & $A$ & $\mathrm{~N}$ & $\mathrm{~N}$ & $\mathrm{~N}$ & $\mathrm{NR}$ & $\mathrm{NR}$ & NR & $\mathrm{R}$ \\
\hline 06 & $A$ & $\mathrm{~A}$ & $\mathrm{~N}$ & $\mathrm{~N}$ & $\mathrm{~N}$ & $\mathrm{~N}$ & $\mathrm{D}$ & NR & $\mathrm{R}$ & NR & $\mathrm{NR}$ \\
\hline 07 & $A$ & $A$ & $\mathrm{~N}$ & $\mathrm{~N}$ & $\mathrm{~N}$ & $\mathrm{~N}$ & $\mathrm{~N}$ & NR & NR & NR & $\mathrm{R}$ \\
\hline 08 & $A$ & $A$ & $\mathrm{~N}$ & $\mathrm{~N}$ & $\mathrm{~N}$ & $\mathrm{~N}$ & $D$ & $\mathrm{NR}$ & $\mathrm{R}$ & NR & $\mathrm{NR}$ \\
\hline 09 & $A$ & $A$ & $\mathrm{~N}$ & $\mathrm{~N}$ & $\mathrm{~N}$ & $\mathrm{~N}$ & $\mathrm{~N}$ & NR & NR & $\mathrm{R}$ & $\mathrm{NR}$ \\
\hline 10 & $A$ & $A$ & $\mathrm{~N}$ & $\mathrm{~N}$ & $\mathrm{~N}$ & $\mathrm{~N}$ & $\mathrm{D}$ & NR & $\mathrm{R}$ & NR & $\mathrm{NR}$ \\
\hline 11 & $A$ & $A$ & $\mathrm{~N}$ & $\mathrm{~N}$ & $\mathrm{~N}$ & $\mathrm{~N}$ & $\mathrm{~N}$ & NR & NR & NR & $\mathrm{R}$ \\
\hline 12 & $A$ & $\mathrm{~A}$ & $\mathrm{~N}$ & $\mathrm{~N}$ & $\mathrm{~N}$ & $\mathrm{~N}$ & $D$ & $\mathrm{NR}$ & $\mathrm{R}$ & NR & $\mathrm{NR}$ \\
\hline 13 & $A$ & $A$ & $\mathrm{~N}$ & $\mathrm{~N}$ & $\mathrm{~N}$ & $\mathrm{~N}$ & $\mathrm{D}$ & NR & $\mathrm{R}$ & NR & $\mathrm{NR}$ \\
\hline 14 & A & $A$ & $A$ & $A$ & $A$ & $A$ & $\mathrm{DC}$ & $\mathrm{NR}$ & NR & NR & $\mathrm{R}$ \\
\hline 15 & $A$ & $\mathrm{~A}$ & $\mathrm{~N}$ & $\mathrm{~N}$ & $\mathrm{~N}$ & $\mathrm{~N}$ & $\mathrm{D}$ & $\mathrm{NR}$ & $\mathrm{R}$ & NR & $\mathrm{NR}$ \\
\hline 16 & $A$ & $A$ & $\mathrm{~N}$ & $\mathrm{~N}$ & $\mathrm{~N}$ & $\mathrm{~N}$ & $\mathrm{~N}$ & NR & NR & NR & $\mathrm{R}$ \\
\hline 17 & $A$ & A & A & $A$ & A & A & $\mathrm{N}$ & NR & NR & NR & $\mathrm{R}$ \\
\hline 18 & $\mathrm{~N}$ & $\mathrm{~A}$ & $\mathrm{~N}$ & $\mathrm{~N}$ & $A$ & $\mathrm{~A}$ & $D$ & $\mathrm{R}$ & NR & NR & $\mathrm{NR}$ \\
\hline 19 & $A$ & $A$ & $\mathrm{~N}$ & $\mathrm{~N}$ & $\mathrm{~N}$ & $\mathrm{~N}$ & $\mathrm{D}$ & NR & $\mathrm{R}$ & NR & $\mathrm{NR}$ \\
\hline 20 & A & A & $\mathrm{N}$ & $\mathrm{N}$ & $\mathrm{N}$ & $\mathrm{N}$ & $\mathrm{N}$ & NR & NR & NR & $\mathrm{R}$ \\
\hline 21 & $A$ & $A$ & $\mathrm{~N}$ & $\mathrm{~N}$ & $\mathrm{~N}$ & $\mathrm{~N}$ & $\mathrm{D}$ & $\mathrm{NR}$ & $\mathrm{R}$ & $\mathrm{NR}$ & $\mathrm{NR}$ \\
\hline 22 & $A$ & $A$ & $\mathrm{~N}$ & $\mathrm{~N}$ & $\mathrm{~N}$ & $\mathrm{~N}$ & $\mathrm{D}$ & NR & $\mathrm{R}$ & NR & $\mathrm{NR}$ \\
\hline 23 & A & $A$ & $\mathrm{~N}$ & $\mathrm{~N}$ & $\mathrm{~N}$ & $\mathrm{~N}$ & $\mathrm{~N}$ & NR & $\mathrm{NR}$ & NR & $\mathrm{R}$ \\
\hline 24 & $A$ & $\mathrm{~A}$ & $\mathrm{~N}$ & $\mathrm{~N}$ & $\mathrm{~N}$ & $\mathrm{~N}$ & $\mathrm{~N}$ & $\mathrm{NR}$ & $\mathrm{R}$ & $\mathrm{NR}$ & $\mathrm{NR}$ \\
\hline 25 & $A$ & $\mathrm{~A}$ & $\mathrm{~N}$ & $\mathrm{~N}$ & $\mathrm{~N}$ & $\mathrm{~N}$ & $\mathrm{D}$ & NR & NR & NR & $\mathrm{R}$ \\
\hline 26 & $A$ & A & $\mathrm{N}$ & $\mathrm{N}$ & $\mathrm{N}$ & $\mathrm{N}$ & $\mathrm{N}$ & $\mathrm{R}$ & NR & NR & $\mathrm{NR}$ \\
\hline 27 & $\mathrm{~A}$ & $A$ & $\mathrm{~N}$ & $\mathrm{~N}$ & $\mathrm{~N}$ & $\mathrm{~N}$ & $\mathrm{D}$ & NR & $\mathrm{NR}$ & $\mathrm{R}$ & $\mathrm{NR}$ \\
\hline 28 & $A$ & $\mathrm{~N}$ & $A$ & $A$ & $\mathrm{~N}$ & $\mathrm{~N}$ & $\mathrm{DC}$ & $\mathrm{NR}$ & $\mathrm{R}$ & NR & NR \\
\hline 29 & A & A & $\mathrm{N}$ & $\mathrm{N}$ & $\mathrm{N}$ & $\mathrm{N}$ & $\mathrm{N}$ & NR & $\mathrm{R}$ & NR & $\mathrm{NR}$ \\
\hline 30 & A & A & $\mathrm{N}$ & $\mathrm{N}$ & $\mathrm{A}$ & A & $\mathrm{N}$ & NR & NR & NR & $\mathrm{R}$ \\
\hline 31 & $A$ & $\mathrm{~A}$ & $\mathrm{~N}$ & $\mathrm{~N}$ & $\mathrm{~N}$ & $\mathrm{~N}$ & $\mathrm{D}$ & $\mathrm{NR}$ & NR & NR & $R$ \\
\hline 32 & A & $A$ & $\mathrm{~N}$ & $\mathrm{~N}$ & $\mathrm{~N}$ & $\mathrm{~N}$ & $\mathrm{~N}$ & NR & NR & NR & $\mathrm{R}$ \\
\hline
\end{tabular}

FA -Fosfatase alcalina

GGT- Gama-glutamilfosfatase

AMIL- Amilase

LIP-Lipase

TGO- Aspartato amino transferase

TGP- Alanina amino transferase

USG - Ultra-sonografia

KEHR- Colangiografia pelo dreno de Kehr
$\mathrm{N}$ - Normal

A- Alterado

R- Realizado

NR- Não realizado

D - Dilatado

DC - Cálculo com dilatação

ECO- Ecoendoscopia

CP-Colangioressonâcia magnética 


\section{PÓS-PROCEDIMENTO}

\begin{tabular}{|c|c|c|c|c|c|}
\hline $\mathbf{N}^{\circ}$ & P.L. & AMIL. & LIP. & COMP. & T.I.P. \\
\hline 01 & $E$ & $\mathrm{~N}$ & $\mathrm{~N}$ & NC & 02 \\
\hline 02 & $\mathrm{E}$ & $\mathrm{N}$ & $\mathrm{N}$ & NC & 02 \\
\hline 03 & DTD & $\mathrm{N}$ & $\mathrm{N}$ & NC & 06 \\
\hline 04 & $E$ & $\mathrm{~N}$ & $\mathrm{~N}$ & NC & 02 \\
\hline 05 & $E$ & $\mathrm{~N}$ & $\mathrm{~N}$ & NC & 02 \\
\hline 06 & $E$ & $\mathrm{~N}$ & $\mathrm{~N}$ & NC & 04 \\
\hline 07 & $E$ & $\mathrm{~N}$ & $\mathrm{~N}$ & NC & 02 \\
\hline 08 & $E$ & $\mathrm{~N}$ & $\mathrm{~N}$ & NC & 02 \\
\hline 09 & $E$ & $\mathrm{~N}$ & $\mathrm{~N}$ & NC & 02 \\
\hline 10 & $\mathrm{E}$ & $\mathrm{N}$ & $\mathrm{N}$ & NC & 01 \\
\hline 11 & DBD & $\mathrm{N}$ & $\mathrm{N}$ & NC & 06 \\
\hline 12 & $E$ & $\mathrm{~N}$ & $\mathrm{~N}$ & $\mathrm{NC}$ & 02 \\
\hline 13 & $E$ & $\mathrm{~N}$ & $\mathrm{~N}$ & $\mathrm{H}$ & 07 \\
\hline 14 & $E$ & $\mathrm{~N}$ & $\mathrm{~N}$ & $\mathrm{NC}$ & 02 \\
\hline 15 & $\mathrm{E}$ & $\mathrm{N}$ & $\mathrm{N}$ & NC & 02 \\
\hline 16 & $E$ & $\mathrm{~N}$ & $\mathrm{~N}$ & NC & 02 \\
\hline 17 & $\mathrm{E}$ & AA & A & $\mathrm{P}$ & 11 \\
\hline 18 & $E$ & $\mathrm{~N}$ & $\mathrm{~N}$ & NC & 02 \\
\hline 19 & $E$ & $\mathrm{~N}$ & $\mathrm{~N}$ & NC & 02 \\
\hline 20 & E-DBD & $\mathrm{N}$ & $\mathrm{N}$ & NC & 10 \\
\hline 21 & DTD & $\mathrm{N}$ & $\mathrm{N}$ & NC & 06 \\
\hline 22 & $E$ & $\mathrm{~N}$ & $\mathrm{~N}$ & NC & 02 \\
\hline 23 & DCD & $\mathrm{N}$ & $\mathrm{N}$ & NC & 06 \\
\hline 24 & $E$ & $\mathrm{~N}$ & $\mathrm{~N}$ & NC & 02 \\
\hline 25 & $E$ & $\mathrm{~N}$ & $\mathrm{~N}$ & NC & 02 \\
\hline 26 & $\mathrm{E}$ & $\mathrm{N}$ & $\mathrm{N}$ & NC & 05 \\
\hline 27 & DTD & $\mathrm{N}$ & $\mathrm{N}$ & $\mathrm{NC}$ & 02 \\
\hline 28 & $E$ & $\mathrm{~N}$ & $\mathrm{~N}$ & NC & 02 \\
\hline 29 & E- DBD & $\mathrm{N}$ & $\mathrm{N}$ & NC & 06 \\
\hline 30 & $E$ & $\mathrm{~N}$ & $\mathrm{~N}$ & NC & 02 \\
\hline 31 & $E$ & AA & A & $\mathrm{P}$ & 07 \\
\hline 32 & $E$ & $\mathrm{~N}$ & $\mathrm{~N}$ & $\mathrm{NC}$ & 02 \\
\hline
\end{tabular}

P.L.-Procedimento realizado

AMIL- amilase

LIP. Lípase

COMP.- complicações

T.I.P.- tempo de internação pós-procedimento

E- DBD- endoscópico sem sucesso - derivação bilio-digestiva

$\mathrm{DBD}=$ Derivação bilio-digestiva

DTD- Derivação trans-duodenal

$P$ - pancreatite

$\mathrm{H}$ - hemorragia

A- aumentado

$\mathrm{N}$ - normal 


\section{ANEXO 02}

\section{PROTOCOLO DE AVALIAÇÃO DE PACIENTES PORTADORES DE COLEDOLITIASE RESIDUAL}

\section{A - IDENTIFICAÇÃO}

Nome:

Pront:

Procedência:

Data Nasc:

Idade:

Estado Civil:

Profissão:

Sexo:

Naturalidade:

Procedência: Zona rural ( ） Zona urbana( ) UF:

Endereço atual:

Cor: Branco ( ) Negro( ) Amarelo ( ) Outros( )

Maneira de estabelecer contato:

Data Inter.

Data de alta:

Dias de Internação: 


\section{B - SINTOMATOLOGIA}

- Emagrecimento

$(\quad) \operatorname{sim}(\quad)$ não

- Plenitude pós-prandial ( ) sim ( ) não

- Má digestão

( ) $\operatorname{sim}(\quad)$ não

- Cólica abdominal Quantos episódios?

- Icterícia Quantos episódios?

Há quanto tempo o ultimo?

- Colúria

) $\operatorname{sim}(\quad)$ não

- Acolia fecal

) $\operatorname{sim}(\quad)$ não

\section{C - EXAMES FÍSICOS}

- Estatura(cm):

Peso:

IMC:

- Estado de nutrição: Bom ( ) Regular ( ) Ruim ( )

\section{I- abdômen}

- Timpanismo

( ) $\operatorname{sim}(\quad)$ não

- Fígado palpável da ( ) sim ( ) não

a $\mathrm{cm}$ da reborda costal

- Baço palpável

- Vesícula palpável

( ) sim

) não

- Sinal de Murphy

( ) sim

) não

- Icterícia

( ) $\operatorname{sim} \quad(\quad)$ não 


\section{II- ANTECEDENTES}

- Colelitíase na família ( ) sim （）não

- Passado de icterícia ( ) sim ( ) não

- Intolerância a alimentos gordurosos ( ) sim ( ) não

- Dor em HD ( ) sim ( ) não

- Cirurgia abdominal ( ) sim ( ) não

- Procedimento sobre as vias biliares ( ) sim ( ) não O que?

Há quanto tempo?

\section{D - EXAMES LABORATORIAIS}

- Hemograma:

- Amilase:

- Lipase:

- Gama GT:

- Fosfatasse alcalina:

Bilirrubinas: TOTAIS:

BD:

BI: 


\section{E - EXAMES DE IMAGEM DIRIGIDOS}

- Colecistograma oral

$(\quad) \operatorname{sim}(\quad)$ não

RESULTADO:

- Colecistograma venoso:

( ) $\operatorname{sim}(\quad)$ não

RESULTADO:

- Ultra-sonografia

( ) $\operatorname{sim} \quad(\quad)$ não

RESULTADO:

- Colangiografia trans-parietohepatica: ( ) sim ( ) não

RESULTADO:

- Tomografia computadorizada: ( ) sim ( ) não

RESULTADO:

- Colangio-ressonância

( ) $\operatorname{sim} \quad(\quad)$ não

RESULTADO:

- Colangio pancreatografia retrogada ( ) sim ( ) não RESULTADO:

- Ecoendoscopia: ( ) sim ( ) não RESULTADO:

\section{F - procedimento realizado}


G - Exames pós-procedimento

- Hemograma:

- Amilase:

- Lípase:

- Blirubinas totais:

$\mathrm{BD}$ :

$\mathrm{Bl}:$

H - complicações pós-procedimento

I - Tempo de internação pós-procedimento(em dias)

J - Observações: 
8. REFERÊNCIAS BIBLIOGRÁFICAS 


\section{8- REFERÊNCIAS BIBLIOGRÁFICAS}

ALLEN B . BERNHOFT $N$. et al Sludge is calcium bilirubinate associated witth bile stasis, Am J surg 141:51, 1981.

ALIPERTI, G.; EDMIJNDGWJCZ, S.A.; SOPER, N.J. et al, Combined endoscopic sphincterotomy and laparoscopiceholecystectomy in patients with choledocholithiasis and cholecystolithiasis. Ann Tnt Med, v. 115, n. 10, P. 783-6, 1991.

AMOUYAL, P.; AMOUYAL, G.; LEVY, P. et al. Diagnosis of choledocholithiasis by endoscopic ultrasonography. Gastroenterology, v. 106, n. 4, p. 1062-7, 1994.

BARKUN, A.N.; BARKUN, J.S.; FRIED, G.M. et al. Useful predictors of bile duct stones in patients undergoing laparoscopic cholecystectomy. Ann Surg, v. 220, n. 1, p.32-9, 1994.

$\mathrm{BERCl}$, G. Preoperative ERCP and intraoperative cholangiography in the age of laparoscopic cholecystectomy. Surg Endosc, v. 7, p. 2, 1993.

BERCI, G.; MORGENSTERN, L. Laparoscopic management of common bile duct stones. Surg Endosc, v. 8, p. 1168-75, 1994. 
BERGMAN, J.J.G.H.M.; MIEY, S.V.D.; RAUWS, E.A.J. et al. Long-term follow-up after endoscopic sphincterotomy for bile duct stones in patients younger than 60 years of age. Gastrointest Endosc, v. 44, n. 6, p. 643-9, 1996.

BERTHOU, J.C.; DRQUARD, F.; CHARBQNEAU, P.; MOUSSALIER, K. Evaluation of laparoscopic management of common bile duct stones in 220 pacients. Surg Endosc, v. 12, p. 16-22, 1998.

BISS K, HO KJ MIKKELSON B, et al :Some unique biologic characteristies of the masai of East Africa. N Engl J Med 284-694, 1971.

BOULAY, J.; SCHELLENBERG, R; BRADY, P. Role of ERCP and therapeutic biliary endoscopy in association with laparoscopic cholecystectomy. Am J Gastroenterol, V.87, n,7, p. 837.42, 1992.

BREFORT, J.L.; SAMANA, G.; LB ROUX, Y.; LANGLOIS, G. Traitement laparoscopique de la lithiase de la voie biliaire principale. Etude de 56 cas. Chirurgie, v. 124, p. 38-44, 1999.

BURCH TA, COMESS LJ, BENNETT PH: The problem of gallbladder diseese among pima indich.PAHO scientifie publication $n^{\circ} 165$ Washington, DC Pan American Health Organization, 1968, p 82.

CARROLL, B.J.; PHILLIPS, E.H.; ROSENTHAL, R. et al. One hundred consecutive laparoscopic cholangiograms. Results and conclusions. Surg Endosc, v. 10, p. 319-23, 1996.

CECCONELLO, I. Contribuição ao conhecimento da histopatologia do colédoco. São Paulo, 1979. Tese ( Mestrado ) Instituto Brasileiro de Estudos e Pesquisas de Gastroenterologia 
CHAMPAULT, G. La lithiase de la voie biliaire principale (VBP) A l'heure de la chirurgie par laparoscopie. Quelle strategie? Ann Chir, v. 47, n. 7, p. 592 7, 1993.

CLASSEN, M.; SAFRANY, L. Endoscopic papillotomy and removal of gallstones. Br Med J, v. 15, p. 371-4, 1975.

COELHO, J.C.U.; BONTLHA, R.; PITAKI, S.A.M. et al. Prevalence of gallstones in a Brazilian population. Int Surg, v. 84, p. 25..8, 1999.

COMFORT, M.W.; GRAY, H.K.; VVILSON, J.M.; The silent gaistone: A ten to twenty year follow up study of 112 cases. Ann Surg, v.128, n.5, p. 931-7, 1948.

COPPOLA, R.; RICCIONI, M.E.; CILETTI, S. et al. Analysis of complications of endoscopic sphincterotomy for biliary stones in a consecutive series of 546 patients. Surg Endosc,v. II,p. 129 32, 1997.

COTTON, RB. Endoscopic management of bile duct stones; (apples and oranges). Gut, V. 25, p. 587-97, 1984.

COTTON, PB. Endoscopic retrograde cholangiopanceratography and laparoscopic cholecystectomy. Am J Surg, v. 165, p. 474-8, 1993.

COTTON, RB.; LEHMAN, G.; VENNES, J. et al. Endoscopic sphincterotomy complications and their management: na attempt at consensus. Gastrointest Endosc, v. 37, n. 3, p. 383-93, 1991.

CREMA, B.; SILVA, A.A,; MARTINS Jr, A. et al. Anastomose colédocoduodenal por laparoscopia no tratamento da coledocolitiase. Boletim da FBG Gastren, edição especial, 1996. 
CRONAN, J.J. US diagnosis of choledocholithiasis; A reappraisial. Radiology, v. 161, p. 133-4, 1986.

CSENDES, A. Common bile duct stones: Introduction. World J Surg, v. 22, p. 1113, 1998.

CSENDES, A.; BURDILES, P.; DJAZ, J.C. Present role of classic open choledochostomy in the surgical treatment of patients with common bile duct stones. World J Surg, v. 22, n. 11, p. 1167-70, 1998./

DANILEWITZ, MD. Early postoperative endoscopic sphincterotomy foe retained common bile duct stones. Gastrointest Endosc, v. 35, n. 4, p. 298 9, 1989.

DAVIS, W.Z.; COTTON, P.B.; ARIAS, R. et al. ERCP and sphincterotomy in the context of laparoscopic cholecystectomy: Academic and community practice patterns and results. Am J Gastroenterol, v. 92, n. 4, p. 597-601, 1997.

DION, Y.; RATElLE, R.; MORIN, J.; GRAVEL, D. Common bile duct exploration:

The place of laparoscopic choledotomy. Surg Laparose Endosc, v. 4, n. 6, p. 419-24, 1994.

DOMENE, C.E.; SANTO, M.A.; VOLPE, P. et al. Laparoscopy choledocoduodenostomy. Hepato-Gastroenterol, v. 45, p. 122, 1998.

DOMENE, C.E.; SANTO, M.A.; VOLPE, P. et al. Laparoscopy treatment of holedocholithiasis. Hepato-Gastroenterol, v. 45, p. 126, $1998 \mathrm{C}$.

EDMUNDOWICZ, S.A. Common bile duct stones. Gastrointest Endose Clin N $\boldsymbol{A m}$, v. 5, n. 4, p. 817-24, 1995. 
ESCUDERO-FABRE, A.; ESCALLON Jr, A.; SACK, J. et al. Choledochoduodenostomy. Analysis of 71 cases followed for 5 to 15 years. Ann Surg, v, 213, n. 6, p. 635-44, 1991.

FERRIS, D.O.; VIBERT, J.C. The common bile duct: Significance of its dimeter. Ann Surg, v.149, n.2, p. 249-5 1, 1959.

FITZGIBBONS, R.J.; RYBERG, A.A.; ULUALP, K.M. et al. An alternative technique for treatment of choledocholithiasis found at laparoscopic cholecystectomy. Arch Surg, v. 130, p. 638-42, 1995.

FLETCHER, D.R. Changes in the practice of biliary surgely and ERCP during the introduction of laparoscopic cholecystectomy to Australia: Their possible significance. Aust N Z J Surg, v. 64, p. 75-80, 1994.

FLETCHER, D.R. Common bile duct calculi at laparoscopic cholecystectomy: A techinique for management. Aust N Z J Surg, v. 63, p. 7104, 1993.

FRAZEE, R.C.; ROBERTS, J.; SYMMOM)S, R. et al. Combined laparoscopic and endoscopic management of cholelithiasis and choledocholithiasis, Am J Surg, v. 166, p. 702 6, 1993.

GAMA-RODRIGUES, J. J.; BRESCIANI, C. Colecistectomia por videolaparoscopia. In:

MINCIS, M., ed. Gastroenterologia e hepatologia. São Paulo, Lemos Editorial, 1997. p.815-826.

GANEY, J.B.; JOHNSON Jr., P,A.; PRILLAMAN, RE,; McSWAIN, \&R. Cholecystectomy: clinical experience with a large series. Am J Surg, v. 151, p. 352-7, 1986. 
GARDNER, A.M.N.; HOLDEN, W.S.; MONKS, P.J.W. Disappearing gallstones. Br J Surg, v. 53, n. 2, p. 114-20, 1966.

GERBER, A.; APT,M.K. The case against routine operative cholangiography. Am. J. Surg., v.143, p. ${ }^{734-6}{ }_{7} 1982$.

GIGOT, J.F.; NAVEZ, B.; ETIENI4E, J. et al. A stratified intraoprerative surgical strategy is mandatory during laparoscopic common bile duct exploration for common bile duct stones. Surg Endosc, v. 11, p. 722-8, 1997.

GRAHAM, S.M.; FLOWERS, J.L.; SCOTT, T.R. et al. Laparoscopic cholecystectomy and common bile duct stones. The utility of planned perioperative endoscopic retrograde cholangiography and sphincterotomy: Experience with 63 patients. Ann Surg, v. 218, n. 1, p. 61-7, 1993.

HAJNSWORTH, P.J.; RHODES, M.; COMPERTZ, R.H.K. et al. Imaging of the common bile duct in patients undergoing laparoscopic cholecystectomy. Gut, v. 35, p. $\quad 991 \sim 5,1994$.

HARDY, KJ. Gallstones and laparoscopic cholecystectomy: A consensus? Med I Aust, v. 160, p. 583-7, 1994.

HIAUER-JENSEN, M.; KARENSEN, R.; NYGAARD, K. et al. Consequences of routine peroperative cholangiography during cholecystectomy for gallstone disease; A prospective, randomized study. World J Surg, v. 10, n. 6, p. 9961002, 1986,

HAUER-JENSEN, M.; KARENSEN, R.; NYGAARD, K. et al. Prospective randomized study of routine intraoperative cholangiography during open cholecystectomy: long-term follow-up and multivariate analysis of predictors of choledocholithiasis. Surgery, v. 113, n. 3, p. 318-23, 1993. 
HIENSMAN, C.; CROSTHWAITE, A,; CUSCHIERJ,A. Transcystic biliary decompression after direct laparoscopic exploration of the common bile duct. Surg Endosc, v.11, p. 1106-10, 1997.

HUGUIER, M.; BORNET, P.; CHARPAK, Y. et al. Selective contraindications based on multivariate analysis for operative cholangiography in biliary lithiasis. Surg Gyn Obst, v. 172, p. 470-4, 1991.

HUNTER, J.G. Laparoscopic transcystic common bile duct exploration. Am J Surg, v. 163, p. 53-8, 1992.

HUNTER, J.G.; SOPER, N.J. Laparoscopic management of bile duct stones. Surg Clin N Am, v. 72, n. 5, p. 1077-97, 1992.

JOHNSON, A.S.; FERRARA, J.J.; STEINBERG, S.M. et al. The role of endoscopic retrograde cholangiopancreatography: Sphincterotomy versus common bile duct exploration as a primary technique in the management of choledocholithiasis. Am Surg, v. 59, n. 2, p. 78-84, 1993.

JONES, D.B.; DUNNEGAN, D.L,; SOPER, N.J. Results of a change to routine fluorocholangiography during laparoscopic cholecystectomy. Surgery, v. 118, n. 4,p. 693 702, 1995.

JOYCE, W.P KEANE, R.; BURKE, G.J. et al. Identification of bile duct stones in patients undergoing laparoscopic cholecystectomy. Br J Surg, v. 78, n. 10, p. 1174-7, 1991.

KAWAI, K.; AKASAKA,Y.; MJJRAKAMI, K. Endoscopic sphincterotomy of the ampula of Vater. Gastrointest. Endosc., V. 20 p. 148-51, 1974

KIM, K.H.; KIM, W.; LEE, H.1. et al. Prediction of common bile duct stones; Its validation in laparoscopic cholecystectomy. Hepato-gastroenterol, v. 44, p. 1574-9, 1997. 
KULLMAN, B.; BORCH, K.; LJNDSTROM, B. et al. Management of bile duct stones in the era of laparoscopic cholecystectomy: Appraisial of routine operative cholangiography and endoscopic treatment. Eur J Surg, V. 162, p. 873-80, 1996.

LACAINE, F.; CORLETh, M.B.; BISMUTH, H. Preoperative evaluation of the risk of common bile duct stones. Arch Surg, v. 115, p. 11 14 6, 1980.

LAI, E,C.S.; MOK, F.P.T.; TAN, E.S.Y. et al. Endoscopic biliary drainage for severe acute cholangitis. N Engi J Med, v. 236, n. 24, p. 1582 6, 1992.

LAMBERT, M.E.; BETTS, C.D.; FARAGHER. E.B. et al. Endoscopic sphincterotomy: the whole truth. Br J Surg, v. 78, p. 473-6, 1991,

LANDONI, N.; CHOPITA, N.; JMELNITZKY, A. Esfinterotolixia endosc6pica complicaciones y seguimiento. Acta Gastroent Latinoamer, v. 23, p. 27-31, 1993.

LEZOCHE, B.; PAGANTNI, A.M. Single-stage laparoscopic treatment of gallstones and common bile duct stones in 120 unselected, consecutive patients. Surg Endosc, v. 9,p.1070-5, 1995.

LEZOCHE, B.; PAGANINI, A.M. Laparoseopic treatment of gallbladder and common bile duct stones: A prospective study. World J Surg, v. 20, p. 53542, 1996.

LEZOCHE, B.; PAGANINI, A.M.; GUERRIERI, M. A new T-tube applier in laparoscopic surgery. Surg Endosc, v. 10, p. 445-8, 1996, JBANORI, LT.; DOMENE, C.E.; NASI, A. et al. Routine laparoscopic cholangiography by a simple and successful techinique. ABCD Arq Bras Cir Dig, v. 10, n. 2,47-50, 1995. 
LIBANORI, I.T.; DOMENI, C.E.; NASI, A. et al. Routini laparoscopic cholangiography by a simple and successful techinique. ABCD Arq Bras Cir Dig, v. 10, n. 2, 47-50, 1995.

LIBERMAN, M.A.; PHILLIPS, ER; CARROLL, B. et at. Cost-effective management of complicated choledocholithiasis; Laparoscopic transcystic duct exploration or endoscopic sphincterotomy. J Am Coil Surg, v. 182, p. 488-94, 1996.

LILLEMOE, K,D.; YEO, C.J.; TALAMiLNI, M.A. et al. Selective cholangiography. Current role in laparoscopic cholecystectomy. Ann Surg, v. 215, n. 6, p. 669-76, 1992.

LORIMER, J.W,; LAUZON, J.; FAIRFULL-SMITH, Ri.; YELLE, J. Management of choledocholithiasis in the time of laparoscopic cholecystectomy. Am J Surg, v, 174,p. 68-71, 1997.

LUND, J. Surgical indications in cholelitiasis: Prophylatic cholecystectomy elucidated on the basis of long-term follow up on 526 nonoperated cases. Ann Surg. v, 151, n, 2,p. 153-61, 1960.

LYGIDAKIS, N.J. The incidence and s'gnificance of common bile duct dilatation in biliary calculous diseases. Word J Surg, v. 8, p. 327-34, 1984.

McENTEE, G.; GRACE, PA.; BOURCHIER-HAYES, D. Laparpscopic cholecystectomy and the common bile duct. Br J Surg, v. 78, p. 385-6, 1991.

MILLAT, B.; FINGERHUT, A.; DELEUZE, H. et al. Proapective evaluation in 121 consecutive unselected patients undergoing laparoscopic treatment of choledocholithiasis. Br J Surg, v. 82, p. 1266-9, 1995. 
MILLER, B.M.; KOZAREK, R.A.; RYAN, J.A. et al. Surgical versus endoscopic management of common bile duct stones. Ann Surg, v. 207, n. 2, p. 135-4 1, 1988.

MILLER, B.M.; KJMMELSTIIEL, F.M.; WINKLER, W.P. Management of common bile duct stones in the era of laparoscopic cholecystectomy. Am $\mathbf{J}$ Surg, v. 169, p. 273-6, 1995.

MIRIZZI, P.L. Operative cholangiography. Surg Gyn Obst, v. 49, p. 702-10, 1937.

MIRIZZI, P.L. Primary suture of the common bile duct in choledocholithiasis. Arch Surg, v. 51, p. 44-54, 1942.

MONTEIRO DA CUNHA, J. B.; PENTEADO, S.; MACHADO, M.C.C.; PINOTTI, H.W. Coledocolitiase. In: PINOTTI, H.W.; CECCONELLO, L; GAMA-RODRIGUES, J.J.; HABR-GAMA, A.; MACHADQ M.C.C.; SAAD,W.A.; FAJNTUCH, J.; DOMLENE, C.E., ed. Tratado de clinica cirúrgica do apareiho digestivo. Sao Paulo, Livraria Atheneu Editora, 1994. p.933-40

MOREAUX, J. Traditional surgical management of common bile duct stones: A prospective study during a 20-year experience. Am J Surg, v. 169, p. 220 6, 1995.

MORGENSTERN, L.; BERCI, G.; PASTERNAK, Eli. Bile leakage after biliaty tract surgery. Surg Endosc, v. 7, p. 432-8, 1993.

MURISON, M.S.C.; GARTELL, P.C.; McGINN, F.P. Does selective peroperative cholangiography result in missed common bile duct stones? $\mathbf{B r}$ J Surg, v. 76, n. 12, p. 1343, 1989. 
NAKAYAMA F, KOGA A; Hepatolithiasis: present status: World J surg 8:9, 1984

NAVARRETE, C,G.; CASTILLO, C.T.; CASTILLO, P.Y. Choledocholithiasis; Percutaneous treatment. World J Surg, v. 22, n. 11, p. 11514, 1998.

NEOPTOLEMOS, J.R; HOFMANN, A.F.; MOOSSA, A.R. Chemical treatment of stones in the biliary tree. Br J Surg, v. 76, n. 7, p. 515-24, 1986.

NEOPTOLEMOS, J.P.; CARR-LOCKE, D.L.; FOSSARD, D.P. Prospective randomised study of operative endoscopic sphincterotomy versus surgery alone for common bile duct stones. Br Med J, v. 294, p. 470-4, 1987.

NEOPTOLEMOS, J.P.; CARR-LOCKE, D.L.; LONDON, N.J. et al. Controlled trial of urgent endoscopic retrograde cholangiopancreatographygraphy and endoscopic sphincterotomy versus conservative treatment for acute pancreatitis due to gallstones. Lancet, v. 29, p. 979-83, 1988.

NEOPTOLEMOS, J.P,; SHAW, D.E.; CARR-LOCKIE, D,L. A multivariate analysis of preoperative risk factors in patients with common bile duct stones. Ann Surg, v.209, n. 2, p.|7 6|, 1989k

NEUHAUS, H,; FEUSSNER, H.; UNGEFEUER, A. et al. Prospective evaluation of the use of endoscopic retrograde cholangiography prior to laparoscopic cholecystectomy. Endoscopy, v. 24, p. 745 9, 1992.

NEUHAUS, C,; SONNENBERO, A.; MUELLER, J. Comparison of extrahepatic bile duct size measured by ultrasound and by different radiographic methods, Gastroenterology, v. 87, n. 3, p. 615-21, 1984.

NIH CONSENSUS CONFERENCE. Gallstones and laparoscopic cholecystectomy. NIH consensus development panel on gallstones and laparoscopic cholecystectomy. JAMA, v. 269, n. 8, p. 1018-24, 1993. 
PAGANINI, A.M.; LEZOCHE, B. Follow-up of 161 unselected consecutive patients treated laparoscopically for common bile duct stones. Surg Endosc, v. 12 , p. 23-9, 1998.

PAVONE, P.; LAGHI, A.; LOMANTO II). et al. MR cholangiography (MRC) in the evaluation of CBD stones before laparoscopic cholecystectomy. Surg Endosc, v. 11,p. 982-5, 1997.

PEREIRA-LIMA, L.; KALIL, A.N. Coledocoduodenostomia intema suprapapilar. Uma altemativa técnica para remoçao de cálculos impactados. Rev Bras Med, v. 15, n. 6, p. 349 51,1988.

PERISSAT, J.; HIJJIBREGTSE, K.; KEANE, F.B.V. et al. Management of bile duct stones in the era of laparoscopic cholecystectomy. Br J Surg, v. 81, p. 799 8 10, 1994.

PHILLIPS, E.H.; CARROLL, B.J.; FALLAS, M.J. Common duct stones: Removal before or during laparoscopic cholecystectomy? Surg Endosc, v. 6, p. 266, 1992.

PHILLIPS, E.H. Controversies in the management of common duct calculi. Surg Clin N Am, v. 74, n, 4, p. 93 1-51, 1994.

PHILLIPS, E.H.; ROSENTHAL, R.J.; CARROLL, B.J.; FALLAS, M.J. Laparoscopic trans-cystic-duct common-bile-duct exploration. Surg Endosc, v. 8, p. 13 89-94, 1994.

PHILLIPS, E,H.; LIBERMAN, M.; CARROLL, B.J et al. Bile duct stones in the laparoscopic era: Is the preoperative sphincterotomy necessary? Arch Surg, v. 130, p.8806, 1995. 
PINOTTI, H.W.; CECCONELLO I.; ZILBERSTEIN, B,; POLLARA, W. M.; Litiase do colédoco. Considerações sobre 200 casos operados. Atual. Cir., V. 7, p. 69-86, 1979.

PINOTTI, H. W.; DOMENE, C. B.; NASI, A.; SANTO, M. A.; LIBANORI, H. T.Colecistectomia laparoscopica e suas perspectivas. In: PINOTTI, H. W.;CECCONELLO, I; GAMA-RODRIGUES, J. J.; HABR-GAMA, A.; MACHADO, M.C. C. SAAD, W. A.; FAINTUCH, J.; DOMENE, C. B., ed. Tratado de clinica cirúrgica do aparelho digestivo. São Paulo, Livraria Atheneu Editora, 1994. v. 2, p. 8\&-93.

PITT, H.A. Role of open choledochotomy in the treatment of choledocholithiasis. Am J Surg, v. 165, p. 483-6, 1993.

RIEGER, R.; WAYAND, W. Yield of prospective, noninvasive evaluation of the ommon bile duct combined with selective ERCP/sphincterotomy in 1390 onsecutive laparoscopic cholecystectomy patients. Gastrointest Endosc, v. 42, n., p. 6-12, 1995.

ROBERTSON, G.S.M.; JAGGER, K.; JOHNSON, P.R.V. et al. Selection criteria for preoperative endoscopic retrograde cholangiopancreatography in the laparoscopic era. Arch Surg, v. 131, p. 89-94, 1996.

ROBINSON, G.; HOLLINSHEAD, J.; FALK, G.; MOULTON, J. Technique and results of laparoscopic choledochotomy for the management of bile duct / Iculi. Aust N Z J Surg, v. 65, p. 347-9, 1995.

ROSENTHAL, R.J.; ROSSI, R.L.; MARTIN, R.F. Options and strategies for the management of choledocholithiasis. World J Surg, v. 22, p. 1125-32, 1998.

SAKAI, P.; ARTIFON, E.L.A.; ISHiOKA, S. et al. Uso de endopr6tese biliar pl stica na coledocolitiase. GED, v. 18, n. 6, p. 233-6, 1999. 
SALMERON, M.; SIMON, J.-F.; HOUDART, R. et al. Endoscopic ultrasonography (BUS) versus invasive methods for the diagnosis of common bile duct stones (CBDS). Gastroenterology, v. 106, n. 4, p. A357, 1994.

SALVIOLI, G.; SALATI, R.; LUGLI, R.; ZANNI, C. Medical treatment of biliary duct stones: effect of ursodeoxycholic acid administration. Gut, v. 24, p. 60914, 1983.

SANTO, M.A. Litíase na via biliar principal análise do tratamento cirúrgico por vídeolaparoscopia . Tese do doutorado - USP-SP.2000.

SANTUCCI, L.; NATALINI, G.; SARPI, L. et al, Selective endoscopic retrograde holangiography and preoperative bile duct stone removal in patients scheduled for aparoscopic cholecystectomy: A prospective study. Am J Gastroenterol, v. 91, n. 7,p.1326-30, 1996,

SAVASSI-ROCHA, P.R.; FERREIIRA, J.T.; DJNIZ, M.T.C. et al. Laparoscopic cholecistectomy in Brazil; Analysis of 33,563 cases. Int Surg, v. 82, p. 20843, 1997.

SCHESINGER, W. H. The unsuspected ductal stone. In: BERCI, G.; CUSCHIERI, A. ed. Bile ducts and bile stones. Philadelphia, W.B. Sauders Company, 1997. p.78-82.

SIEGEL, J.H. Biliary tract diseases in the elderly: management and outcome. Gut, v. 41, p. 433-5, 1997.

SINISGALLI, L.A.M.; CHERVIN, E.L.N.; SINISGALLI Jr, CA et al. Treatment of biliary lithiasis by extracorporeal lithotripsy. ABCD Arq Bras Cir Dig, v. 8, n. 4, p. 96-101, 1993. 
SINISGALLI, L.A.M.; CHERVIN, E.L.N.; CI\{ERWJN, E.L.N.; et al. Tratamento da coledocolitiase no serviço de cirurgia do HNSL. IV Congresso Bras de VideoCirurgia da SOBRACIIL, Anais dos Congressos, p. 85, 1999.

SMITH, P.C.; CLAYMAN, RN.; SOPER, N.J. Laparoscopic cholecystectomy and choledochoscopy for the treatment of cholelithiasis and choledocholithiasis. Surgery, v. 111, n. 2, p. 230-3, 1992.

SOPER, N.J. Intraoperative screening for common bile duct stones. Ultrasound or cholangiography? Surg Endosc, v. 12, p. 362, 1998.

SPERANZINI, M.B.; CASTILLO NETTO, J.M.; LIMA Jr, S.E. Defeitos na drenagem cir(irgica do colédoco. Rev Col Bras Cir, v. 20, n. 6, p. 283-8, 1993.

STAIN, S.C.; MASRI, L.S.; FROES, E.T. et al. Laparoscopic cholecystectomT Laboratory predictors of choledocholithiasis. Am Surg, v. 60, p. 767-71, 1994.

STARITZ, P.; PORALLA, T.; DORMEYER, H.; MEYER, K.; BUSCHENFELDE, K.M. Endoscopic removal of common bile duct stones through the intact papilla after medical sphincter dilation. Gastroenterology, v. 88, n. 6, p. 1807-11, 1985.

STIGMANN, G.V.; GOFF, J.S.; MANSOUR, A. et al. Precholecystectomy endoscopic cholangiography and stone removal is not superior to cholecystectomy, cholangiography, and common bile duct exploration. Am J Surg, v. 163, p. 227-30, 1992.

STOKER, M.E. Common bole duct exploration in the era of laparoscopic surgery. Arch Surg, v. 130, p. 265-9, 1995. 
STRASBERG, S.M.; CALLERY, M.P.; SOPER, N.J. Laparoscopic surgery of the bile ducts. Gastrointest Endosc Clin North Am, v. 6, n. 1, p. 8

SOURICK, B.; WASHINGTON, M.; GHAZI, A. Endoscopic retrograde cholangiopancreatography in conjunction with laparoscopic cholecystectomy. Surg Endosc, v. 7, p. 388-92, 1993..

WATERS, G.S.; CRIST, D.W.; DAVOUDI, M. et al. Management of choledocholithiasis encountered during laparoscopic cholecystectomy. Am Surg, v. 62, n. 4, p. 256-8, 1996.

WAY, L.W.; ADMIRAND, W.H.; DUNPHY, J.E. Management of choledocholithiasis, Ann Surg, v. 176, n. 3, p. 347-59, 1981.

WENCKERT, A,; ROBERTSON, B. The natural course of gallstone disease. Eleven-year rewiew of 781 nonoperated cases. Gastroenterology, v. 50, n.3, p. 376-8 1, 1966. 